\title{
Gemini Observations of Galaxies in Rich Early Environments (GOGREEN) I: survey description
}

\author{
Michael L. Balogh, ${ }^{1 \star}$ David G. Gilbank, ${ }^{2,3}$ Adam Muzzin, ${ }^{4}$ Gregory Rudnick, ${ }^{5}$ \\ Michael C. Cooper, ${ }^{6}$ Chris Lidman, ${ }^{7}$ Andrea Biviano, ${ }^{8}$ Ricardo Demarco, ${ }^{9}$ \\ Sean L. McGee, ${ }^{10}$ Julie B. Nantais, ${ }^{11}$ Allison Noble, ${ }^{12}$ Lyndsay Old, ${ }^{13}$ Gillian Wilson, ${ }^{14}$ \\ Howard K. C. Yee, ${ }^{13}$ Callum Bellhouse,,${ }^{10,15}$ Pierluigi Cerulo, ${ }^{9}$ Jeffrey Chan, ${ }^{14}$ \\ Irene Pintos-Castro, ${ }^{13}$ Rane Simpson, ${ }^{1}$ Remco F. J. van der Burg, ${ }^{16}$ Dennis Zaritsky, ${ }^{17}$ \\ Felicia Ziparo, ${ }^{10}$ María Victoria Alonso, ${ }^{18}$ Richard G. Bower, ${ }^{19,20}$ Gabriella De Lucia, ${ }^{8}$ \\ Alexis Finoguenov, ${ }^{21,22}$ Diego Garcia Lambas, ${ }^{18}$ Hernan Muriel, ${ }^{18}$ Laura C. Parker, ${ }^{23}$ \\ Alessandro Rettura, ${ }^{24}$ Carlos Valotto ${ }^{18}$ and Andrew Wetzel $^{25,26,27} \dagger$
}

Affiliations are listed at the end of the paper

Accepted 2017 May 31. Received 2017 May 29; in original form 2017 May 3

\begin{abstract}
We describe a new Large Program in progress on the Gemini North and South telescopes: Gemini Observations of Galaxies in Rich Early Environments (GOGREEN). This is an imaging and deep spectroscopic survey of 21 galaxy systems at $1<z<1.5$, selected to span a factor $>10$ in halo mass. The scientific objectives include measuring the role of environment in the evolution of low-mass galaxies, and measuring the dynamics and stellar contents of their host haloes. The targets are selected from the SpARCS, SPT, COSMOS, and SXDS surveys, to be the evolutionary counterparts of today's clusters and groups. The new red-sensitive Hamamatsu detectors on GMOS, coupled with the nod-and-shuffle sky subtraction, allow simultaneous wavelength coverage over $\lambda \sim 0.6-1.05 \mu \mathrm{m}$, and this enables a homogeneous and statistically complete redshift survey of galaxies of all types. The spectroscopic sample targets galaxies with $\mathrm{AB}$ magnitudes $z^{\prime}<24.25$ and [3.6] $\mu \mathrm{m}<22.5$, and is therefore statistically complete for stellar masses $M_{*} \gtrsim 10^{10.3} \mathrm{M}_{\odot}$, for all galaxy types and over the entire redshift range. Deep, multiwavelength imaging has been acquired over larger fields for most systems, spanning $u$ through $K$, in addition to deep IRAC imaging at $3.6 \mu \mathrm{m}$. The spectroscopy is $\sim 50$ per cent complete as of semester 17A, and we anticipate a final sample of $\sim 500$ new cluster members. Combined with existing spectroscopy on the brighter galaxies from GCLASS, SPT, and other sources, GOGREEN will be a large legacy cluster and field galaxy sample at this redshift that spectroscopically covers a wide range in stellar mass, halo mass, and clustercentric radius.
\end{abstract}

Key words: galaxies: clusters: general-galaxies: evolution.

\section{INTRODUCTION}

Galaxy clusters are extraordinarily valuable as laboratories for a wide range of tests and experiments. They play a central role in studies of cosmology (e.g. Carlberg et al. 1996; Wang \& Steinhardt 1998; Haiman, Mohr \& Holder 2001; Sehgal et al. 2011;

^E-mail: mbalogh@uwaterloo.ca

$\dagger$ Caltech-Carnegie Fellow.
Benson et al. 2013), galaxy and structure formation (e.g. Dressler 1980; Butcher \& Oemler 1984; Balogh et al. 1999b; Ellingson et al. 2001; van den Bosch et al. 2008; Peng et al. 2012; Wetzel et al. 2013), high-energy physics (e.g. Mohr, Mathiesen \& Evrard 1999; Tozzi \& Norman 2001; Pfrommer \& Enßlin 2004; Bialek, Evrard \& Mohr 2001; Balogh, Babul \& Patton 1999a; Cavagnolo et al. 2008; Fabian et al. 2000; Aharonian et al. 2017), supermassive black hole growth (e.g. McNamara \& Nulsen 2007; Alexander \& Hickox 2012), and for the determination of the nature of dark matter (e.g. Clowe et al. 2006; Randall et al. 2008; Bradač et al. 2008; Jee 
et al. 2012). Their enormous gravitational potentials allow them to act as cosmic 'calorimeters', maintaining an observable record of all the energy inputs and outputs associated with galaxy formation over the history of the Universe (e.g. Voit \& Bryan 2001; Gonzalez, Zaritsky \& Zabludoff 2007; Balogh et al. 2008; Vikhlinin et al. 2006; Puchwein, Sijacki \& Springel 2008). They host the most massive galaxies, whose stars are among the first to form (e.g. Lidman et al. 2012; Lin et al. 2013; Marchesini et al. 2014; Fassbender et al. 2014). Clusters are also the ideal places to study rare and extraordinary perturbations to galaxy evolution, such as hydrodynamic stripping of gas (e.g. Vollmer et al. 2000; Merluzzi et al. 2013; Boselli et al. 2016), tidal stripping of matter (Natarajan, Kneib \& Smail 2002), and high-speed gravitational encounters. Much of what we have learned about galaxy evolution is thanks to years of research on these systems.

At $z>1$, when the gas accretion rates, relative gas masses, and star formation rates (SFRs) of galaxies were much higher than they are today, the interactions between galaxies and their environments are also expected to be very different. Large spectroscopic samples have now been built up in clusters approaching $z \sim 1$ (Muzzin et al. 2012; Balogh et al. 2014; Bayliss et al. 2016), but little is known about the physical properties of typical galaxies in $z>1$ clusters. Those spectroscopic studies that do exist are generally restricted to the most massive galaxies (e.g. Snyder et al. 2012; Lotz et al. 2013) or emission-line galaxies (e.g. Zeimann et al. 2013; Nanayakkara et al. 2016) in the most massive clusters (e.g. Nantais et al. 2013; Martini et al. 2013; Stanford et al. 2014). Studying the faint, and more common, galaxy population at $1<z<1.5$ usually relies heavily on photometric redshifts (e.g. Nantais et al. 2016).

Thus for the first round of Gemini Large and Long Programs in 2014, we proposed an ambitious distant cluster legacy survey, titled Gemini Observations of Galaxies in Rich Early ENvironments (GOGREEN), using the GMOS instruments on the North and South telescopes. GMOS has several capabilities that make it ideally suited to studies of galaxy clusters at $z \sim 1$. The nod-and-shuffle (n\&s) mode (Glazebrook \& Bland-Hawthorn 2001) allows excellent sky subtraction at red wavelengths, resulting in much greater efficiency for faint galaxies (as exploited by the GDDS ${ }^{1}$; Abraham et al. 2004). Moreover, the n\&s microslits are up to three times smaller than normal slits, allowing them to be placed with a very high surface density. Together with the new Hamamatsu detectors, which have good sensitivity up to $\lambda \sim 1.05 \mu \mathrm{m}$, GMOS spectroscopy of faint objects is now feasible at $1.0<z<1.5$.

The objective of the survey is to build directly on the work we have done with Gemini in constructing the GCLASS $^{2}$ cluster (Muzzin et al. 2012) and GEEC $2^{3}$ group (Balogh et al. 2014) surveys. GCLASS was a GMOS survey consisting of $\sim 450$ spectroscopically confirmed members of 10 massive clusters at $0.86<z<$ 1.34. Among other things, this enabled new insight into the environmental transformation of galaxies (Muzzin et al. 2014; Foltz et al. 2015; Noble et al. 2013, 2016), the stellar mass content and distribution in clusters (van der Burg et al. 2013, 2014), cluster dynamics (Biviano et al. 2016), and growth of the brightest cluster galaxies (Lidman et al. 2012). An independent but highly complementary survey was GEEC2, which used a sample of 10 X-ray-detected groups in the COSMOS field to address similar questions of galaxy evolution (Balogh et al. 2011; Mok et al. 2013, 2014) and group

\footnotetext{
${ }^{1}$ Gemini Deep Deep Survey.

${ }^{2}$ Gemini CLuster Astrophysics Spectroscopic Survey.

${ }^{3}$ Galaxy Environment Evolution Collaboration 2.
}

dynamics (Hou et al. 2013). The combination of GEEC2 and GCLASS provides a sample that spans more than two orders of magnitude in halo mass, allowing the measurement of halo mass effects on the environmental quenching measurement (Balogh et al. 2016).

GOGREEN uses a similar strategy to extend these works to 1.0 $<z<1.5$ with comparably dense spectroscopy on 21 systems spanning a wide range in halo mass. An important feature of GOGREEN is that our systems are chosen to be representative of the progenitors of today's clusters; this is complementary to efforts focused on the most massive clusters at high redshift, which have few, if any, local descendants. The survey will obtain spectroscopy on a large sample of very faint targets, $z^{\prime}<24.25$ and [3.6] $<22.5$ to obtain a sample of confirmed cluster members, measure cluster dynamics and galaxy stellar populations, and provide critical calibration of photometric redshifts. The survey design is driven by three key science goals, and an aim to provide a legacy data set that is useful to the broader community. These goals are described in more detail below.

\subsection{Environmental quenching and growth of the stellar mass function}

Despite a solid theoretical foundation for the gravitational growth of dark matter structure, galaxy formation models have great difficulty simultaneously reproducing the rate of decline in global SFR, the mass dependence of this decline, and the star formation histories of satellite galaxies (Bower, Benson \& Crain 2012; Weinmann et al. 2012; Hirschmann et al. 2014; De Lucia et al. 2012; Henriques et al. 2015; Genel et al. 2014; Trayford et al. 2015). These problems may be related, as they are all sensitive to assumptions about how gas accretion, ejection and heating processes depend on epoch, environment, and halo mass (McGee, Bower \& Balogh 2014). The conventional picture of the interaction between galaxies and their surroundings is that galaxies enter dense environments with a reservoir of gas (either in the stellar disc, or the halo), and that star formation declines as this reservoir is removed (e.g. Balogh, Navarro \& Morris 2000; Bower et al. 2006; Schawinski et al. 2014; Fillingham et al. 2015). However, cosmological simulations show that galaxies grow as a result of continuous infall from surrounding filaments (e.g. Kereš et al. 2005), a scenario that is supported by indirect observational arguments (e.g. Davé, Finlator \& Oppenheimer 2012; Lilly et al. 2013). While a reservoir may play a role at low redshift, at higher redshift the supply of fresh gas fully dominates over the consumption of the reservoir. This change leads to a prediction that dense environments shut down star formation even more rapidly at $z>1$ than at low redshift (McGee et al. 2014; Balogh et al. 2016; van de Voort et al. 2017). The sensitivity of the observed galaxy population to gas accretion and outflow rates on large scales allows us to use trends with environment to put constraints on feedback and accretion models that may be relevant to the evolution of all galaxies.

Simple but powerful indicators of SFR suppression (or 'quenching') are the evolution of the quiescent galaxy stellar mass function, and the stellar mass dependence of the quiescent fraction (e.g. van den Bosch et al. 2008; Peng et al. 2010; Fillingham et al. 2016; Balogh et al. 2016). From these measurements alone, it is possible to put strong constraints on the quenching time-scale and its evolution (e.g. Tinker \& Wetzel 2010), which is a powerful indicator of how gas-supply and removal mechanisms change with time (McGee et al. 2014; Balogh et al. 2016; Fossati et al. 2017). In the field population, the quiescent galaxy mass function evolves rapidly, 
as star formation is shut down first in the most massive galaxies, and later in dwarfs (e.g. Muzzin et al. 2013a). In massive clusters, this is clearly seen as the growth of the 'red sequence' (e.g. De Lucia et al. 2004; Tanaka et al. 2005; Gilbank \& Balogh 2008; Rudnick et al. 2009, 2012). At $z<1$, most models predict many more lowmass, quiescent galaxies than are observed, a consequence of the well-established overquenching problem (Weinmann et al. 2011; Hirschmann et al. 2014; De Lucia et al. 2012; Henriques et al. 2015; Genel et al. 2014; Trayford et al. 2015).

The situation is much less clear at higher redshift (e.g. Nantais et al. 2016), where the gas content, accretion rates, and SFRs of galaxies are so much higher, and even galaxies in cluster cores have only been satellites for a few Gyr. Moreover, the higher average SFR of field galaxies, and the increased rate at which they are accreted by the cluster, translates directly into a much higher fraction of galaxies observed in the 'transition phase' between actively star forming and quiescent (Poggianti et al. 2009; Mok et al. 2014). Exceptional sensitivity to the galaxy transformation time-scale can be obtained from fairly straightforward modelling of the radial gradients and projected phase space distribution of such subpopulations, compared with the quiescent and star-forming galaxies (e.g. Balogh et al. 2000; Ellingson et al. 2001; McGee et al. 2009; Noble et al. 2013; Taranu et al. 2014; Muzzin et al. 2014; Haines et al. 2015).

GOGREEN is designed specifically to measure the quiescent fraction of galaxies at $1.0<z<1.5$, over a factor $>10$ in halo mass, with a spectroscopic sample statistically complete for all galaxy types down to stellar masses of $M_{*} \sim 10^{10.3} \mathrm{M}_{\odot}$. In addition to the targeted clusters and groups, the survey will result in a comparably-sized field sample selected in the same way. The deep, multiwavelength imaging ensures a robust and homogeneous separation of passive from star-forming galaxies, and a photometric redshift catalogue that is essential to account for the spatial incompleteness of the spectroscopic sample. When complete, the total spectroscopic sample size, including bright galaxy spectroscopy from GCLASS and other published catalogues, will be comprised of $\sim 1000$ cluster members; about half of these will be newly acquired via GOGREEN. This will ensure that statistical uncertainties on the quenched fraction, in bins of stellar and halo mass, are small enough to distinguish between different physical models as described in Balogh et al. (2016).

\subsection{The hierarchical assembly of baryons}

It is a fundamental prediction of $\Lambda$ cold dark matter $(\Lambda \mathrm{CDM})$ theory that massive clusters are built from haloes of lower mass: groups and isolated galaxies (e.g. Berrier et al. 2009; De Lucia et al. 2012; Bullock, Kravtsov \& Weinberg 2001). Since it is difficult to preferentially remove stars from dark matter dominated systems, when these systems merge the fraction of total mass in stars can only increase (via star formation) or remain constant. Therefore, measurement of the stellar fraction, gas fraction, and SFR in haloes of a given mass provide one of the closest possible links between galaxies and this basic prediction of the $\Lambda \mathrm{CDM}$ theoretical framework (Kravtsov, Nagai \& Vikhlinin 2005; Gonzalez, Zaritsky \& Zabludoff 2007; Balogh et al. 2008; Giodini et al. 2009; Gonzalez et al. 2013; Leauthaud et al. 2011, 2012). Precision measurements of this type are essential for calibrating and constraining models, and are an essential complement to abundance-matching or halo occupation distribution model approaches (e.g. Behroozi, Wechsler \& Conroy 2013).

With GOGREEN, we will directly measure the central and total stellar mass of haloes at $1.0<z<1.5$. The spatial and dynami- cal distribution of cluster galaxies is sensitive not only to the field accretion rate, but also to the dynamical friction time and galaxy merger and disruption time-scales. These rates are not well understood theoretically (e.g. De Lucia et al. 2010), despite being primarily gravitational processes, and observations of these distributions provide valuable constraints, as we have shown with GCLASS (e.g. van der Burg et al. 2014).

\subsection{Cluster dynamics and halo masses}

At low redshift, the total mass content and distribution of galaxy clusters can be estimated by gravitational lensing, from the properties of the intracluster plasma under the assumption of hydrostatic equilibrium, or from the distribution and kinematics of cluster galaxies. The latter method always provides critical independent information from the other two, and is especially important for clusters at high redshifts, which are notoriously difficult to detect by their $\mathrm{X}$-ray emission or weak-lensing signal. At $z>1.0$, our knowledge of the mass profiles of galaxy clusters is therefore limited to only a few individual clusters.

Dynamical analyses of nearby clusters have shown their $M(r)$ to be well characterized by either an NFW (Navarro, Frenk \& White 1997) or an Einasto, Kaasik \& Saar (1974) profile, passive galaxy orbits to be isotropic and star-forming galaxy orbits to be radially elongated (e.g. Biviano \& Girardi 2003; Biviano \& Katgert 2004). The NFW and Einasto models appear to also fit well the $M(r)$ of $z \sim 0.6$ clusters, but the orbits of passive galaxies evolve with $z$, and at $z \sim 0.6$ are more similar to those of star-forming galaxies (Biviano \& Poggianti 2009; Biviano et al. 2013).

Using a stack of $\sim 400$ galaxies in 10 clusters of the GCLASS sample, Biviano et al. (2016) have shown that the $M(r)$ of $z \sim 1$ clusters is still well described by the NFW model, with a concentration as predicted by numerical simulations, and that the orbits of passive and star-forming cluster galaxies are indistinguishable and mildly radially elongated. With GOGREEN we will trace this evolution to $z \sim 1.5$. We will be able to measure whether the NFW and Einasto models remain valid representations of the cluster $M(r)$, which is particularly interesting as the onset of dynamical equilibrium in galaxy clusters is still a poorly understood process (e.g. Dehnen \& McLaughlin 2005). Combined with the velocity anisotropy profile $\beta(r)$, we can measure the more fundamental pseudo-phase space profile (Dehnen \& McLaughlin 2005; Lapi \& Cavaliere 2009). Evolution in this profile can distinguish between cluster assembly via fast, violent relaxation processes, and smooth accretion of matter from the field (Hansen et al. 2009).

\subsection{Legacy science}

GOGREEN will provide deep, multiwavelength imaging, and spectroscopy over 21 systems spanning a factor $>10$ in halo mass. Future surveys like eRosita, Euclid and LSST will find large samples of high-redshift clusters. These surveys rely on spectroscopic studies to calibrate their observable quantitites in way that is necessary for cosmological applications. The depth and completeness of GOGREEN spectroscopy is a good complement to efforts like Bayliss et al. (2016) and Stanford et al. (2014), which aim to sparsely sample relatively massive galaxies in a much larger set of clusters.

A byproduct of our survey will be a deep spectroscopic field survey of $>600$ galaxies at $1.0<z<1.5$, with homogeneous and well-understood selection criteria. At present, none of the existing wide-field spectroscopic surveys have the red sensitivity to match GOGREEN depth at $1.3<z<1.5$. Claims about evolution in the 
stellar mass function and star formation history, for example are based on photometric redshifts, which are notoriously unreliable in regions of parameter space where spectroscopic calibration is unavailable. The GOGREEN field survey will be twice the size of GDDS (Abraham et al. 2004), and 0.5 mag deeper, allowing an unparalleled spectroscopic measurement of the galaxy mass function, separated by galaxy type. It will provide a crucial calibration sample for photometric redshifts out to $z=1.5$, needed by surveys like LSST and PanStarrs.

In this paper, we describe the survey design (Section 2), spectroscopic observations (Section 3), and the current status of the project (Section 4). All magnitudes reported in this paper are on the AB system.

\section{SURVEY DESIGN}

\subsection{Objectives}

GOGREEN is designed primarily to learn about the stellar populations in galaxies that inhabit massive haloes, $M \gtrsim 5 \times 10^{13} \mathrm{M}_{\odot}$, at $1.0<z<1.5$. To do this, it is essential to cover a large range in both stellar mass and halo mass. In particular, it is important to study low stellar mass galaxies, which are rarely quenched in the field population. At $1.0<z<1.5$, such galaxies are faint and red, making it challenging to even obtain a redshift since most strong absorption features are redshifted to wavelengths where night sky emission lines are strong. To take advantage of the range in halo mass, it is important to be able to characterize those haloes, which, in part, requires redshifts for as many cluster members as possible, including the brightest ones. These two goals - very deep spectroscopy of faint galaxies, together with a large number of redshifts for bright galaxies - are difficult to achieve, and most cluster surveys aim to do one or the other. GOGREEN is specifically designed to achieve both goals within the same program.

\subsection{Cluster sample}

GOGREEN is constructed to enable robust measurements of the populations and dynamics of cluster members at $1.0<z<1.5$, as a function of clustercentric radius and stellar mass. The greatest power of the survey will come from combining the sample with comparable data on lower redshift systems, such as EDisCS (White et al. 2005), MeNEACS (Sand et al. 2012), CCCP (Hoekstra et al. 2012), CNOC (Yee, Ellingson \& Carlberg 1996), GEEC (Wilman et al. 2005; McGee et al. 2011), and CLASH (Postman et al. 2012; Rosati et al. 2014). These surveys cover a halo mass range $\sim 10^{13} \mathrm{M}_{\odot} \sim 5 \times$ $10^{15} \mathrm{M}_{\odot}$, for $z<1$. In order to sample the antecedents of the lower redshift systems, we select galaxy systems in three approximate bins of richness: groups $\left(M<10^{14} \mathrm{M}_{\odot}\right)$, typical clusters $\left(10^{14}<\right.$ $\left.M / \mathrm{M}_{\odot}<5 \times 10^{14}\right)$, and very massive clusters $\left(M>5 \times 10^{14} \mathrm{M}_{\odot}\right)$. The initial focus of our spectroscopy is on the typical and massive clusters, with the groups at a lower priority until we are assured the total time available is not unduly compromised by weather loss.

For the cluster sample, it is natural and efficient to build on the existing investment in GCLASS (Muzzin et al. 2012), so we include five GCLASS clusters at $z>1$ for much deeper follow-up spectroscopy. These clusters were themselves selected from SpARCS (Wilson et al. 2009; Muzzin et al. 2009; Demarco et al. 2010b), a survey that identified clusters based on overdensities of 'redsequence' galaxies (e.g. Gladders \& Yee 2000) using shallow $z$ ' and IRAC $3.6 \mu \mathrm{m}$ images over $42 \mathrm{deg}^{2}$. In addition to the five GCLASS clusters, we also include the next richest systems within the target
Table 1. The table presents the 21 galaxy clusters and groups in the GOGREEN sample, in ordered by RA within three mass classes. Redshifts are given in column (4); values in parentheses are estimates. The final column gives the $\left(z^{\prime}-[3.6]\right)$ colour of the identified red sequence, used in mask design (see Section 2.4.3).

\begin{tabular}{llll}
\hline Name & RA \\
& Dec. & $z$ & $\left(z^{\prime}-[3.6]\right)_{\mathrm{RS}}$ \\
$(\mathrm{AB})$
\end{tabular}

\begin{tabular}{lrrrl}
\hline \multicolumn{5}{c}{ Massive SPT clusters } \\
SPT-CL J0205-5829 & 31.43900 & -58.48290 & 1.320 & 2.61 \\
SPT-CL J0546-5345 & 86.65616 & -53.75800 & 1.067 & 2.01 \\
SPT-CL J2106-5844 & 316.51912 & -58.74110 & 1.132 & 2.17 \\
\multicolumn{5}{c}{ SpARCS clusters } \\
SpARCS0035-4312 & 8.95708 & -43.20678 & 1.335 & 3.0 \\
SpARCS0219-0531 & 34.93156 & -5.52494 & $(1.3)$ & 2.5 \\
SpARCS0335-2929 & 53.76487 & -29.48219 & 1.368 & 3.1 \\
SpARCS1033+5753 & 158.35650 & 57.89000 & 1.455 & 3.0 \\
SpARCS1034+5818 & 158.70599 & 58.30917 & $(1.4)$ & 3.0 \\
SpARCS1051+5818 & 162.79680 & 58.30087 & 1.035 & 1.9 \\
SpARCS1616+5545 & 244.17180 & 55.75714 & 1.156 & 2.2 \\
SpARCS1634+4021 & 248.64751 & 40.36433 & 1.177 & 2.3 \\
SpARCS1638+4038 & 249.71517 & 40.64525 & 1.196 & 2.5 \\
& \multicolumn{5}{c}{ Groups } & & \\
SXDF60XGG & 34.18937 & -5.16353 & 1.410 & 3.29 \\
SXDF64XGG & 34.32375 & -5.17140 & 1.030 & 2.41 \\
SXDF87XGG & 34.52729 & -5.05699 & 1.402 & 2.81 \\
SXDF49XGG & 34.53474 & -5.07140 & 1.059 & 2.20 \\
SXDF76XGG & 34.74128 & -5.32334 & $(1.4)$ & 3.01 \\
COSMOS-28 & 149.45758 & 1.67241 & 1.258 & 2.5 \\
COSMOS-63 & 150.35332 & 1.93337 & 1.234 & 2.45 \\
COSMOS-221 & 150.57024 & 2.49864 & 1.146 & 2.41 \\
COSMOS-125 & 150.62720 & 2.15920 & $(1.45)$ & 3.21 \\
\hline & & & &
\end{tabular}

redshift range; these are expected to be comparable to, or slightly less massive than, the GCLASS systems.

To sample the most massive clusters, we include three spectroscopically confirmed clusters detected via their Sunyaev-Zeldovich signature from the South Pole Telescope (SPT) survey. Like the GCLASS sample, the SPT clusters have existing spectroscopy available on the brighter galaxies (Brodwin et al. 2010; Foley et al. 2011; Stalder et al. 2013), so GOGREEN is primarily targeting the fainter objects.

For the groups, we selected nine X-ray-detected systems from the COSMOS and Subaru-XMM Deep Survey (SXDS) fields, in an analogous way to the selection made for GEEC2 (Balogh et al. 2014). Deep, multiwavelength imaging and exquisite photometric redshifts already exist for these systems, enabling efficient targeting. The COSMOS and SXDS groups are selected from updated versions of the catalogues described in Finoguenov et al. $(2010,2007)$ and George et al. (2011). For target selection in COSMOS, we use the UltraVISTA photometric catalogues of Muzzin et al. (2013b). For SXDS, we use an updated version of the UDS catalogues from Williams et al. (2009) and Quadri et al. (2012), kindly provided by R. Quadri.

The coordinates and redshifts of the 21 systems selected are given in Table 1. We select the targets to ensure they are reasonably distributed in redshift between $1.0<z<1.5$, and in RA and Dec. for efficient observability from Gemini North and South. 
Table 2. The GMOS $z^{\prime}$-band images acquired as part of GOGREEN are described in this table. Images taken on GMOS-N, with the older EEV detector (GN/EEV in column 3), required longer integration times to accommodate the lower sensitivity, compared with the Hamamatsu detectors on GMOS-S (GS/Ham). Depths in column (6) are based on analysis of the Gemini standard pre-imaging pipeline reduction. Position angles (column 5) are chosen to ensure appropriate guide star availability for the MOS follow up. Notes: (1) Saturated pixels alter the background level across the amplifier. (2) Image quality affected by poor active optics correction.

\begin{tabular}{|c|c|c|c|c|c|c|}
\hline Target & Date & $\begin{array}{l}\text { Telescope/ } \\
\text { detector }\end{array}$ & $\begin{array}{l}\text { Integration } \\
\text { time }(\mathrm{ks})\end{array}$ & $\begin{array}{l}\text { PA } \\
(\text { deg })\end{array}$ & $\begin{array}{l}\text { Depth } \\
5 \sigma(\mathrm{AB})\end{array}$ & $\begin{array}{l}\text { Conditions/ } \\
\text { notes }\end{array}$ \\
\hline SPT0205 & 2014 September 28 & GS/Ham & 5.4 & 90 & 25.2 & IQ $0.7,(1)$ \\
\hline SPT2106 & 2015 April 11 & GS/Ham & 5.4 & 100 & 24.1 & IQ $0{ }^{\prime} 6,(1)$ \\
\hline SpARCS0035 & 2014 September 28 & GS/Ham & 5.4 & 0 & 24.75 & IQ $0.65,(1)$ \\
\hline SpARCS0219 & 2014 October 14 & GS/Ham & 5.4 & 0 & 25.0 & IQ $0.65,(1)$ \\
\hline SpARCS0335 & 2014 September 28 & GS/Ham & 5.4 & 185 & 25.6 & IQ $0.65,(1)$ \\
\hline SpARCS1034 & 2015 March 28 & GN/EEV & 8.91 & 90 & 25.4 & IQ $0 ! 7$ \\
\hline SpARCS1051 & 2015 May 8 and 14 & GN/EEV & 8.91 & 90 & 25.1 & IQ $0 . .8$ \\
\hline SpARCS 1616 & 2015 May $14-15$ & GN/EEV & 8.91 & 0 & 25.6 & IQ $0 . .8$ \\
\hline SpARCS1634 & 2015 May 14 & GN/EEV & 5.22 & 215 & 25.7 & IQ 0.7 \\
\hline SpARCS1638 & 2015 May $14-15$ & GN/EEV & 8.91 & 90 & 25.6 & IQ 0.7 \\
\hline
\end{tabular}

\subsection{Multiwavelength imaging}

We obtained deep $z^{\prime}$-band imaging on our 12 massive cluster targets, using GMOS-N (EEV) and GMOS-S (Hamamatsu) detectors, at the start of our program (end of 2014). The nine group targets already have sufficiently deep $z^{\prime}$-band data for spectroscopic target selection from COSMOS and SXDS. The GMOS observations are described in Table 2. GMOS-S observations, which were taken with the red-sensitive Hamamatsu detectors, were typically taken with integration times of $1.5 \mathrm{~h}$. For the northern systems, integration times were typically $2.5 \mathrm{~h}$, to account for the lower sensitivity of the EEV detector. There is some variation in these times to account for differences in observing conditions. Most systems were observed under 70 percentile seeing conditions $\left(\sim 0^{\prime} .7\right.$ in $\left.z^{\prime}\right), 70$ percentile cloud cover (up to $\sim 0.3$ mag extinction), and 80 percentile sky brightness. A $3 \times 3$ dither grid pattern was executed, with 6 " steps.

\subsubsection{GMOS z' band}

The GMOS $z^{\prime}$-band data were reduced using the Gemini IRAF packages and standard procedures, including fringe correction. Before 2015 May, saturated pixels on the detector would affect the background level along the entire row of that amplifier. An example is shown in Fig. 1, for SpARCS0035. This is primarily a cosmetic nuisance, but does eliminate a small fraction of the detector area from spectroscopic follow up.

Zero-points for the imaging were determined by comparing with pre-existing, but shallower, $z^{\prime}$ imaging from SpARCS (CanadaFrance-Hawaii Telescope, CFHT/MegaCAM) and the SPT collaboration (CTIO/MOSAIC-II). The SpARCS zero-points were obtained from standard stars taken during night time observations in the CFHT queue; the zero-points are applied during the initial reduction stages by TERAPIX. We note that the GMOS $z^{\prime}$ band (particularly for the GMOS-S Hamamatsu chips) has a different wavelength coverage than the $z^{\prime}$ band on most cameras. This is because while the transmission of the $z^{\prime}$-band filter itself typically extends up to $1.3 \mu \mathrm{m}$, the effective wavelength is set by the declining quantum efficiency of the chips being used. For most cameras, the transmission at $\lambda>9500 \AA$ is negligible. Both the deep depletion EEV chips and the Hamamatsu chips used here are more red-sensitive than typical CCDs, extending past $10000 \AA$, and therefore the effective wavelength of the $z^{\prime}$ band is longer. However, a direct comparison of

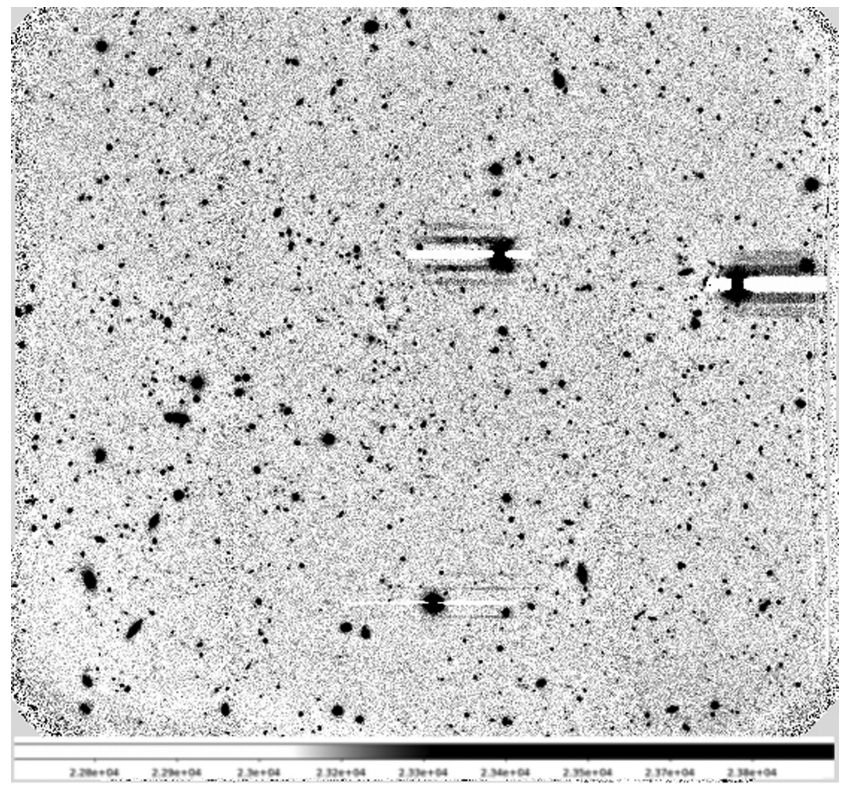

Figure 1. The GMOS-S $z^{\prime}$-band image of SpARCS0035, highlighting the variable background across an amplifier in the presence of saturated stars. The problem was fixed when the video board was replaced in 2015 May.

$z^{\prime}$ magnitudes taken with Gemini-S Hamamatsu and CFHT shows no significant offset, relative to the photometric uncertainties, as a function of magnitude. We therefore neglect any colour term in the photometry.

Magnitude limits are determined from the rms of 10 pixel (1".6) blank-sky aperture measurements across the field. Preliminary $5 \sigma$ limits determined this way are given in Table 2.

\subsubsection{Spitzer IRAC imaging}

All but three of our clusters have publicly available deep ( $5 \sigma$ depth of at least $2 \mu \mathrm{Jy}$, or $\mathrm{AB}=23.1$ ) [3.6] $\mu \mathrm{m}$ imaging from Spitzer IRAC. Most of the data come from SERVS (Mauduit et al. 2012), S-COSMOS (Sanders et al. 2007) and SpUDS (PI: J. Dunlop, as described in Galametz et al. 2013). The three SPT clusters were 
Table 3. Deep imaging observations of the SpARCS and SPT clusters in our sample, as of semester 2017A. Table entries indicate instruments from which imaging data have been obtained, or for which observations are scheduled. These are GMOS $\left(z^{\prime}\right)$, VIMOS (UBVRIz) on the VLT, Suprimecam (SC) and HyperSuprimeCam (HSC) on Subaru $($ griz $Y)$, Fourstar on Magellan $\left(J_{1} J K_{s}\right)$, HAWK-I on VLT $\left(Y J K_{s}\right)$, and WIRCAM on CFHT $\left(Y J K_{s}\right)$. Target depths are indicated in the column headings, and listed observations are expected to be close to those depths. We do not list a target depth for $u / U$ as this (non-critical) band is more heterogeneous. Imaging is mostly complete apart from the northern clusters (SpARCS $10 \mathrm{~h}$ and $16 \mathrm{~h}$ clusters) for which $J$ and $K_{S}$ observations are still required. All targets also have deep Spitzer IRAC imaging with depths of at least 23.1 at $3.6 \mu \mathrm{m}$. Entries marked with a ${ }^{\star}$ have been scheduled as of the time of writing.

\begin{tabular}{|c|c|c|c|c|c|c|c|c|}
\hline $\begin{array}{l}\text { Cluster } \\
\text { Target depth }\end{array}$ & $u / U$ & $\begin{array}{c}g / B / V \\
26.5\end{array}$ & $\begin{array}{c}r / R \\
26.5\end{array}$ & $\begin{array}{c}i / I \\
25.0\end{array}$ & $\begin{array}{l}z^{\prime} / z \\
26.0\end{array}$ & $\begin{array}{l}\mathrm{Y} / \mathrm{J} 1 \\
24.5\end{array}$ & $\begin{array}{c}J \\
24.0\end{array}$ & $\begin{array}{c}K_{s} \\
23.5\end{array}$ \\
\hline SpARCS0035 & VIMOS & VIMOS & VIMOS & VIMOS & GMOS/VIMOS & Fourstar & HAWK-I & HAWK-I \\
\hline SpARCS0219 & VIMOS & VIMOS & VIMOS & VIMOS & GMOS/VIMOS & Fourstar & Fourstar & Fourstar \\
\hline SpARCS0335 & VIMOS & VIMOS & VIMOS & VIMOS & GMOS/VIMOS & HAWK-I & Fourstar & Fourstar \\
\hline SPT0546 & VIMOS & VIMOS & VIMOS & VIMOS & GMOS/VIMOS & Fourstar & Fourstar & Fourstar \\
\hline SpARCS1051 & Megacam & $\mathrm{SC}$ & $\mathrm{SC}$ & $\mathrm{SC}$ & GMOS/HSC & HSC & & \\
\hline SpARCS1616 & Megacam & $\mathrm{SC}$ & $\mathrm{SC}$ & $\mathrm{SC}$ & GMOS/HSC ${ }^{\star}$ & $\mathrm{HSC}^{\star}$ & & \\
\hline SpARCS1634 & Megacam & SC & SC & SC & GMOS/HSC $^{\star}$ & $\mathrm{HSC}^{\star}$ & WIRCAM & WIRCAM \\
\hline SpARCS1638 & Megacam & $\mathrm{SC}$ & $\mathrm{SC}$ & $\mathrm{SC}$ & GMOS/HSC ${ }^{\star}$ & $\mathrm{HSC}^{\star}$ & & \\
\hline SPT2106 & VIMOS & VIMOS & VIMOS & VIMOS & GMOS/VIMOS & Fourstar & Fourstar & HAWK-I \\
\hline
\end{tabular}

observed as PI programmes (PI: Brodwin, from programme ID 70053 and 60099).

The remaining three clusters only had imaging from SWIRE (Lonsdale et al. 2003), which has a $5 \sigma$ depth of $7 \mu \mathrm{Jy}$, sufficient only for the brighter targets in our sample. We therefore obtained $1200 \mathrm{~s} \mathrm{pixel}^{-1}$ integrations over $3 \times 2$ maps at [3.6] $\mu \mathrm{m}$ and [4.5] $\mu \mathrm{m}$ from Cycle 13 (PI: McGee, GO programme 13046).

\subsubsection{Other optical and near-infrared imaging}

Multiwavelength imaging is required both to quantify the spectroscopic completeness and to determine the stellar masses and star formation histories of our galaxies. In particular, broad wavelength coverage is crucial for classifying galaxies from their rest-frame colours. We also require good photometric redshifts to understand the relevant completeness for cluster members (e.g. van der Burg et al. 2013). Well-calibrated photometric redshifts will allow us to determine membership at radii outside of those probed by our GMOS spectroscopy.

The nine group targets have existing deep, multiwavelength imaging spanning the full optical-near-infrared (NIR) spectrum from COSMOS and SXDS, and our goal is to obtain comparably deep coverage in the same bands (ugrizYJK) for the other systems. To this end, we have been using available resources to obtain homogeneous imaging on all systems. The current status is described in Table 3. Through observations on VLT, Magellan, Subaru, and CFHT, we expect to have obtained all required data except $J K_{s}$ for the northern systems, by the end of semester 17A. Fig. 2 shows a typical field layout for two fields, SPT0205 in the south and SpARCS1034 in the north.

\subsection{Spectroscopy}

To obtain even low-quality ( $\mathrm{S} / \mathrm{N} \sim 2$ per $\AA$ ) spectroscopy on very faint $\left(z^{\prime} \sim 24\right)$ galaxies requires exposure times of $\sim 15 \mathrm{~h}$ with GMOS. To simultaneously achieve high completeness at brighter magnitudes, we observe each cluster with multiple slit masks, spread over several semesters. Typically 25-30 slits can be assigned to priority targets on each mask, owing to geometrical constraints. We allocate $\sim 15$ of the faintest galaxies $\left(23.5<z^{\prime}<24.25\right)$ to every mask, such that they obtain $15 \mathrm{~h}$ of total integration time. Another 5-10 slits per mask on brighter galaxies are different for each mask. For massive clusters in which we have little or no existing data, we observe six masks of $3 \mathrm{~h}$ each, to maximize the number of brighter targets. By spreading the masks over three semesters we can make adjustments between observations; for example by replacing faint targets that have reached the desired signal-to-noise ratio $(\mathrm{S} / \mathrm{N})$ prematurely. Most of the SpARCS clusters already have extensive spectroscopy from the GCLASS program; for these we plan only four masks of $5 \mathrm{~h}$ each, focusing on the fainter galaxies.

The groups are significantly less rich, and there are fewer bright candidate members. Therefore, we plan only three masks on each, with $5 \mathrm{~h}$ exposures.

\subsubsection{Spectroscopic selection catalogues}

Spectroscopic targets are selected directly from our deep $z^{\prime}-$ band imaging, described in Section 2.3.1. Target selection (see Section 2.4.3) is made using simple magnitude and colour cuts from combined $z^{\prime}$-band and IRAC $3.6 \mu \mathrm{m}$ photometry. Fig. 3 shows an example of our deep imaging in these two bands for SpARCS1634, compared with the original CFHT image from which the cluster was detected in SpARCS.

Photometric catalogues for the spectroscopic selection were made following the methods laid out in Muzzin et al. $(2008,2009)$ and Wilson et al. (2009) for the SpARCS survey, and we refer the reader to those papers for full details. In brief, for a given cluster, objects were detected separately in both the $z^{\prime}$ band and $3.6 \mu \mathrm{m}$ using the SEXTRACTOR package (Bertin \& Arnouts 1996). Detection in separate filters has the advantage of being able to easily flag sources blended in the IRAC images, as well as being able to detect both extremely red and blue objects not detected in complementary filters.

Photometry in the $z^{\prime}$ band was performed in a fixed aperture of 3.' 66 radius, which is chosen as a multiple of the IRAC native pixel scale (1".22), and the SEXTRACTOR mag_auto value was also recorded as the total $z^{\prime}$-band magnitude. The $3.6 \mu \mathrm{m}$ filter photometry was performed in multiple fixed apertures ranging from 3".66 to $244^{\prime \prime} 0$ radius. The total IRAC magnitude was calculated using the method of Lacy et al. (2005), which effectively uses as the total magnitude 

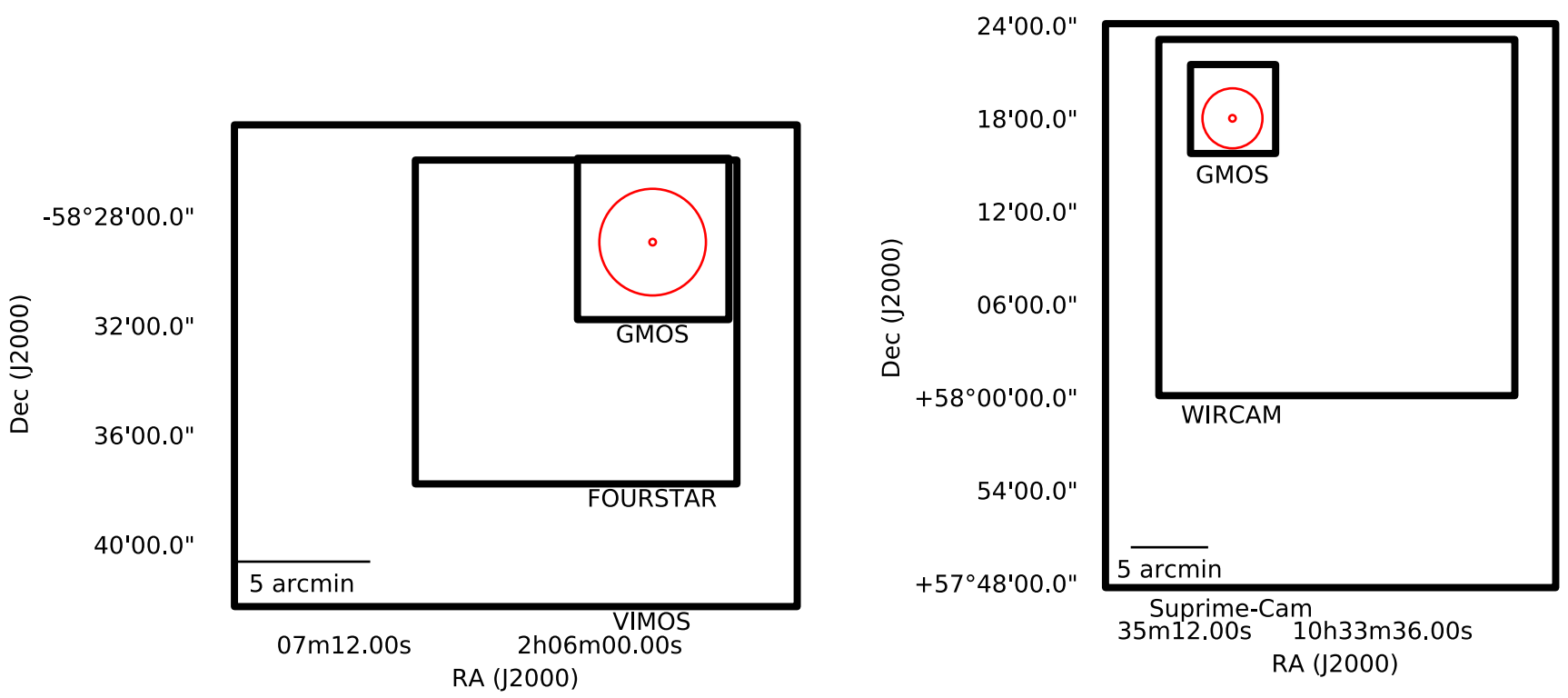

Figure 2. Examples of the imaging footprints for typical clusters, SPT-0205 (left-hand panel) and SpARCS1034 (right-hand panel). The smaller circle marks the cluster location and the larger circle is $1 \mathrm{Mpc}$ (physical) at the cluster redshift, $z=1.32$ and 1.40 respectively. The rectangles (from smallest to largest) show the imaging fields of GMOS, FOURSTAR, and VIMOS (left) and GMOS, WIRCAM, and Suprime-Cam (right). For the four chip mosaic cameras (FOURSTAR, WIRCAM, HAWK-I, and VIMOS), the cluster has been placed at the centre of one quadrant to ensure the full exposure depth is reached around the cluster centre in a single chip. The gaps in these mosaic cameras result in a region of lower exposure time in a central cross of the image.

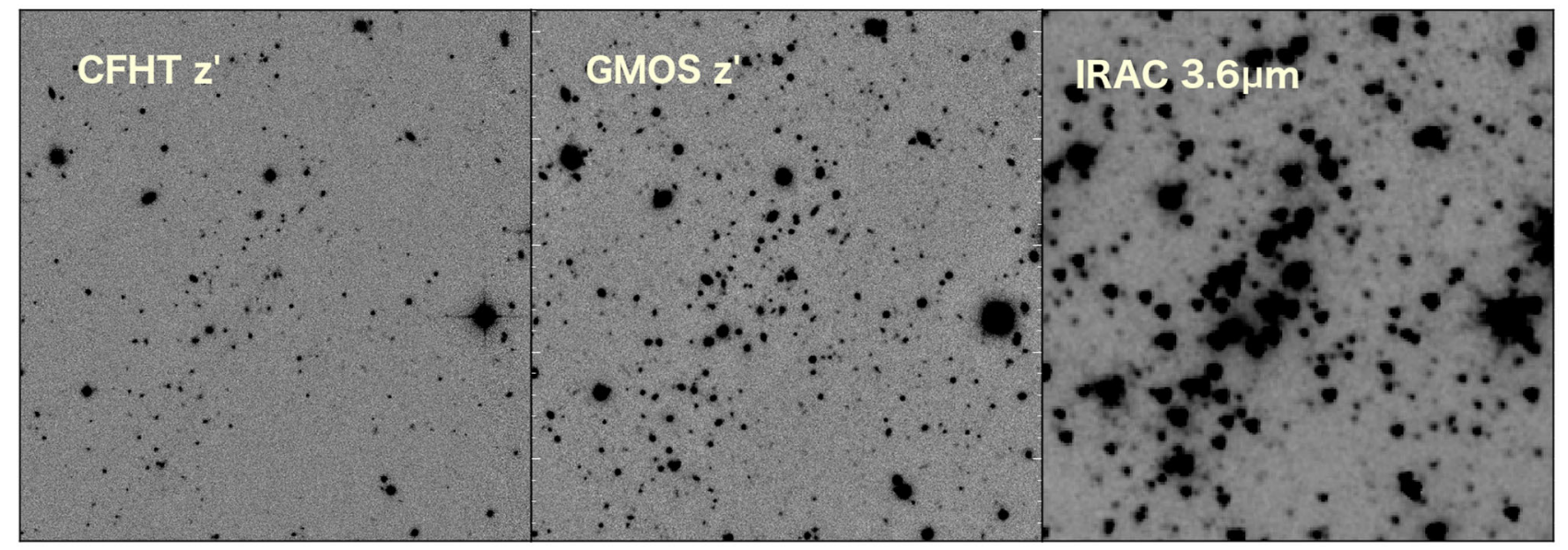

Figure 3. An example of our imaging is shown, for SpARCS1634. The left image shows the central 2'.4 of the original SpARCS imaging available from CFHT MegaCam, from which the clusters were detected. In the middle panel, we present our new $z^{\prime}$ image from GMOS, and on the right, we show the deep IRAC image. All images are oriented with north up and east to the left.

the magnitude measured in the fixed aperture that is closest in size to the estimated isophotal radius of the galaxy as determined with SEXTRACTOR.

Once objects are detected and fluxes measured in each band, objects are matched using a tolerance of $1^{\prime \prime} .0$. This is smaller than the full width at half-maximum (FWHM) of the $3.6 \mu \mathrm{m}$ data, and therefore minimizes the number of spurious matches (e.g. Lacy et al. 2005). Muzzin et al. (2008) estimated that this induces a spurious match rate of $\sim 4$ per cent, most of which are caused by blended sources where the IRAC centroid is misplaced. Therefore, we note that there may be catastrophic photometry for as many as 4 per cent of sources in the selection catalogues. However, since it is caused by random blends primarily of foreground/background galaxies, this should not bias the selection of spectroscopic GOGREEN targets.
We emphasize that these methods are only used for constructing catalogues for selecting spectroscopic targets. Future multiwavelength catalogues will use point spread function (PSF) matching and fitting techniques to mitigate the effect of blending (e.g. van der Burg et al. 2013).

The $z^{\prime}-3.6 \mu \mathrm{m}$ colours are measured using the 3". 66 radius apertures, with an aperture correction for the flux lost from the nonGaussian wings of the IRAC PSF (Lacy et al. 2005). PSF homogenization is not done for the colours because degradation of the deep $z^{\prime}$-band image quality $(\sim 0.7)$ to the poorer image quality of the IRAC data $\left(\sim 1^{\prime \prime} .8\right)$ would cause significant blending and affect the colour measurements. The 3". 66 radius aperture is larger than the FWHM in both $z^{\prime}$ band and $3.6 \mu \mathrm{m}$, and larger than the typical size of high-redshift galaxies $\left(\sim 1^{\prime \prime} .0\right)$ and therefore provides 
an unbiased colour without the need for PSF homogenization, at the sacrifice of some S/N. This method for photometry was used extensively in SpARCS (e.g. Muzzin et al. 2009) and other wide-field Spitzer surveys (e.g. Eisenhardt et al. 2004) and has been shown to provide reliable colours. For all clusters, a clear red sequence is visible (see Sections 2.4.3 and 4), which gives confidence that the photometry is of sufficient quality to select spectroscopic targets.

Stars are identified in the $z^{\prime}$ band using the SEXTRACTOR class_star parameter. This is important both for marking potential mask alignment stars and telluric standards, and for avoiding selecting stars as science targets.

\subsubsection{Instrument configuration}

Spectroscopy is obtained with the GMOS-S and GMOS-N instruments, which cover a 5.5 × 5.5 field of view. All observations on GMOS-S were obtained with the Hamamatsu detector array, which consists of three chips. Two of these have enhanced red response, while the chip at the blue end has enhanced blue response. Pixels are $15 \mu \mathrm{m}$ on a side, corresponding to $0 . ' 080$ pixel $^{-1}$. All our observations are obtained with the detector binned $2 \times 2$, resulting in a pixel scale of 0 "' 16 . On GMOS-N, observations prior to 2017 were obtained with an array of identical EEV deep depletion detectors. These detectors have a pixel scale of 0.0727; as with the GMOS$S$ data we bin $\times 2$ for a final pixel scale of $0^{\prime \prime} 145$. In 2017 , the GMOS-N detector was replaced with a Hamamatsu array identical to the one on GMOS-S.

We observe all fields with the R150 grating, in n\&s mode. The low resolution is chosen to maximize the wavelength coverage on the detector, ensuring that redshift completeness is high. With the $2 \times 2$ detector binning, the dispersion is $3.9 \AA$ for the Hamamatsu detectors (GMOS-S), and $3.5 \AA$ for the EEV (GMOS-N). Slits are 1 '/ wide, resulting in a spectral resolution of $\sim 460$, or $\sim 20 \AA$.

The slits are $3^{\prime \prime}$ long, and we centre the object $0^{\prime} .725$ away from the centre of the slit. The telescope is then nodded by 1 1.45, placing the object 0 '.725 on the other side of the centre. Most of our masks are observed in microshuffle mode, where charge is shuffled by a little more than a slit width. For most clusters we also observe a mask in band-shuffle mode, where the charge is shuffled by a third of the detector height. This is done to achieve maximum target density in the core of the rich clusters.

Fig. 4 shows how our wide wavelength range enables coverage of key spectral features, from the $\mathrm{Mg}$ II absorption line at $2800 \AA$ to the $G$ band at $4300 \AA$, over the full redshift range $1.0<z<1.5$.

We use spectral dithers, observing each mask at three different central grating settings ( 8300,8500 , and $8700 \AA$ ). This allows contiguous wavelength coverage in the presence of chip gaps and bad columns on the detector.

\subsubsection{Spectroscopic target selection and mask design}

Galaxy targets are selected based on their $3.6 \mu \mathrm{m}$ and $z^{\prime}$-band flux from deep IRAC and GMOS imaging. Specifically, galaxies must have total magnitudes [3.6] $<22.5$ and $z^{\prime}<24.25$. To target this range efficiently requires some colour pre-selection to remove foreground and background galaxies. We take different approaches for the 12 clusters (SpARCS and SPT systems), which are fairly rich but lack photometric redshifts, and for the nine groups which are poor but have the advantage of exquisite photometric redshifts.

For the cluster samples, we use a colour cut for avoiding lowredshift $(z<1)$ contamination. This is determined by examining the colour-magnitude distribution of galaxies in UltraVISTA with good

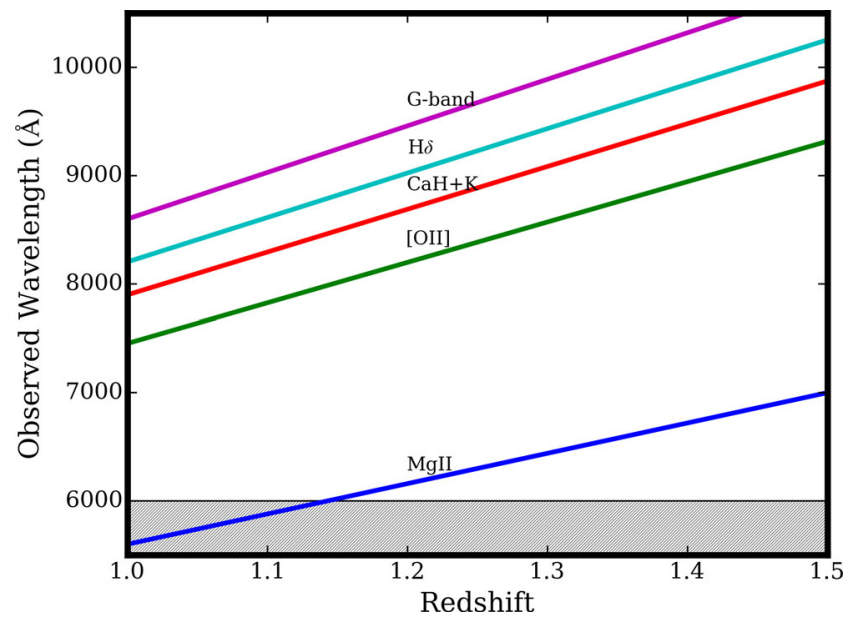

Figure 4. The coverage of key spectral features is shown as a function of redshift, over the target redshift range $0<z<1.5$. While good data are obtained over the full-wavelength range 5500-10 $500 \AA$, the wavelength calibration is unreliable below $6000 \AA$, indicated as the shaded region. With the good red sensitivity and accurate sky subtraction, we are able to identify the usual UV and optical line indices over the full redshift range.

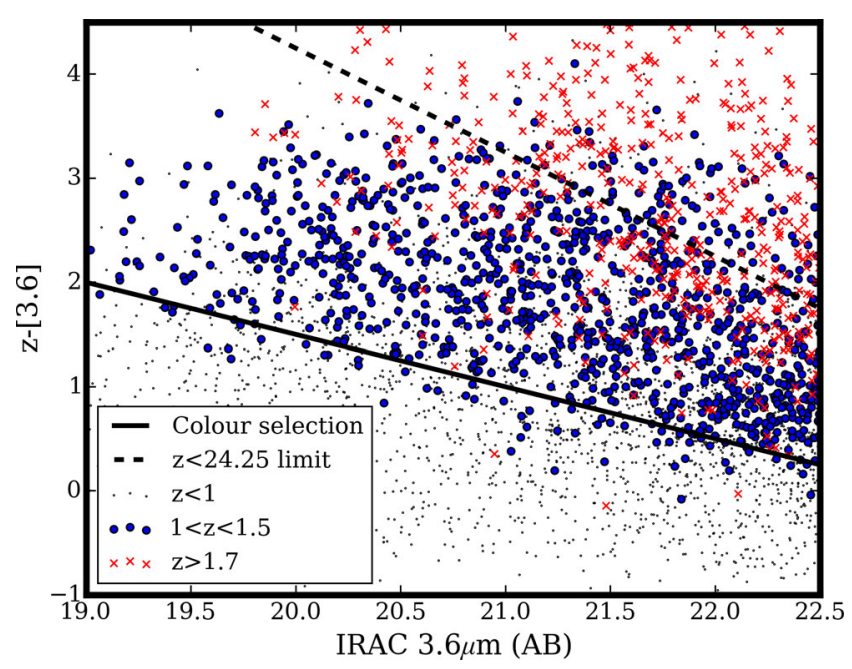

Figure 5. $(z-[3.6])$ as a function of [3.6] magnitude for a random subset of UltraVISTA data (Muzzin et al. 2013b), coloured by photometric redshift. Targets are selected to be redder than the solid black line, to remove contamination from $z<1$ galaxies. The $z^{\prime}$-band limit of the survey, $z^{\prime}<$ 24.25 , is shown by the dashed line and naturally excludes most high-redshift galaxies. Galaxies with $z>1.7$ are shown by red crosses, and will generally not get a measured redshift because [O II] $] \lambda 3727$ is shifted out of our wavelength range.

photometric redshifts (Muzzin et al. 2013b). A random subset of this sample is shown in Fig. 5, with different symbols representing galaxies at $z<1,1.0<z<1.5$, and $z>1.7$. The latter will have the [O II] emission line redshifted beyond our wavelength range and thus we are unlikely to be able to measure a redshift. A simple colour cut $\left(z^{\prime}-[3.6]\right)>2-0.5([3.6]-19)$ is made to exclude low-redshift galaxies. The effectiveness of these cuts is shown in Fig. 6. The solid black line shows the expected fraction of all primary targets that lie in the desired redshift range $1.0<z<1.5$ in an average patch of UltraVISTA. This is $\sim 40$ per cent, roughly independent of magnitude. However, our target fields are not average patches, 


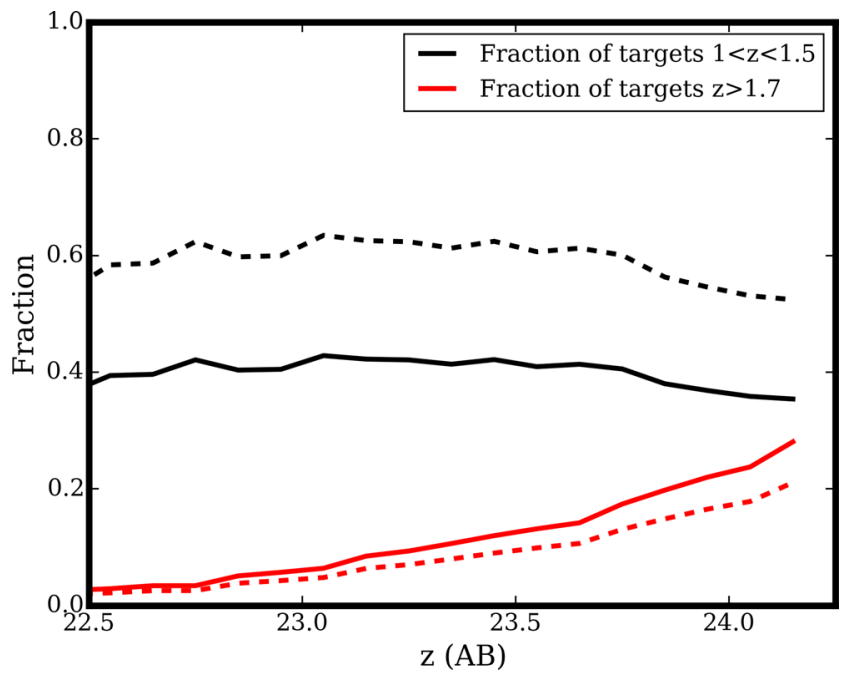

Figure 6. The expected success of our colour selection is shown as a function of total $z^{\prime}$ magnitude. The solid black line shows the fraction of targeted galaxies that are expected to lie in the redshift range $1.0<z<$ 1.5, based on the UltraVISTA (Muzzin et al. 2013b) photometric redshift sample. The red line shows the fraction of targeted galaxies expected to lie at $z>1.7$, for which it is unlikely that we would be able to obtain a redshift. These fractions are derived from a field average, while our targeted areas have massive clusters in the target redshift range. If we assume the $1.0<z<$ 1.5 slice distributed over the GMOS field of view is moderately overdense by a factor $\sim 2$, we obtain the dashed lines. Thus, we expect about 50 per cent of our targets to lie in the required redshift range, with only $\sim 20$ per cent high-redshift contamination at the faintest magnitudes.

but host massive clusters; thus our success rate is expected to be significantly higher than that. The dashed line shows the result if the field is overdense in the $1.0<z<1.5$ redshift slice by a modest factor of two, and this raises the efficiency to $\sim 60$ per cent. The red lines show the fraction of $z>1.7$ galaxies that will be targeted; this rises to at most $\sim 30$ per cent at the faintest magnitudes, and only $\sim 20$ per cent in the presence of an overdense region. The blue colour cut shown in Fig. 6 excludes about 7 per cent of $1.0<z<$ 1.5 galaxies at $z^{\prime}<24.25$ and [3.6] $<22.5$.

For the mask design, in addition to the broad cuts described above, we fit the red sequence in $z^{\prime}-$ [3.6] colour, with a slope of zero. An initial estimate of the colour is made based on the redshift of the cluster and the models of Bruzual \& Charlot (2003) as described in Muzzin et al. (2009). When necessary, this is adjusted based on the overdensity of galaxies on the colour-magnitude diagram. The adopted colours are given in Table 1 . Only galaxies up to $0.2 \mathrm{mag}$ redder than this red sequence are considered primary targets.

In order to optimize the mask design, we then use a Monte Carlo technique, whereby the complete set (3-6 masks) is designed together, and 1000 realizations of each set is performed. The overall aim of the design is to obtain high numbers of galaxies in the bright $(z<23.5)$ and faint $\left(z^{\prime}>23.5\right)$ bins, and ensure reasonable completeness in the cluster core where geometry maximally constrains slit placement and would otherwise lead to underrepresented galaxies simply due to slit collisions. In order to do this, we use the following figure of merit (FOM) to evaluate the mask designs:

$\mathrm{FOM}= \begin{cases}0.5 n_{\mathrm{f}}+0.05 n_{\mathrm{b}}, & \text { if } n_{\mathrm{f}}<11 \\ 0.2 n_{\mathrm{f}}+1.0 n_{\mathrm{b}}, & \text { otherwise }\end{cases}$

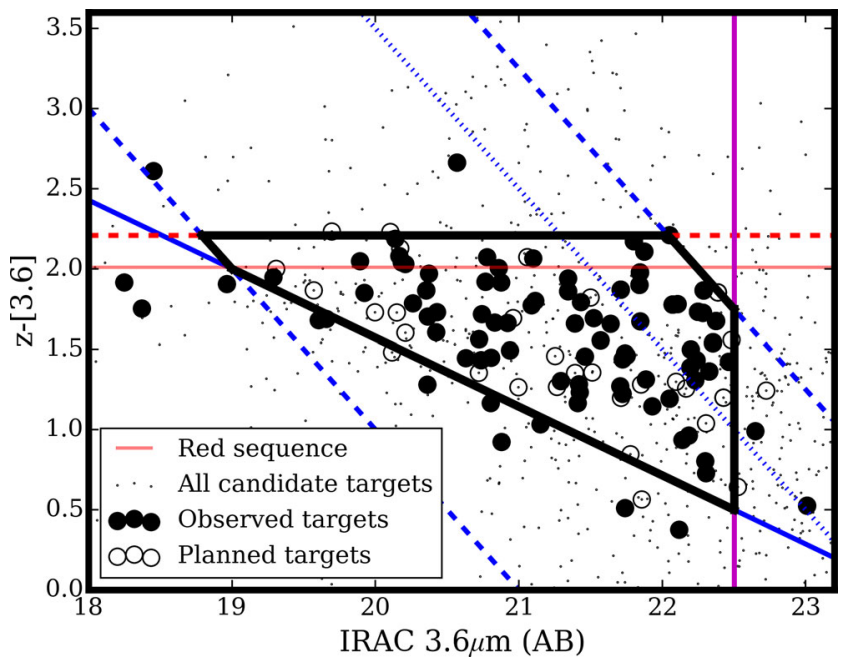

Figure 7. The colour-magnitude diagram of SPT0546 is shown, with all galaxies detected in $z^{\prime}$ and IRAC within the GMOS field of view shown as small dots. The thick black lines outline the selection area for our primary target sample. This is bounded by the IRAC limit of 22.5 (solid, vertical magenta line), the $21<z^{\prime}<24.5$ limits (thick, blue dashed lines), a colour cut to exclude foreground galaxies (thick blue solid line), and a cut 0.2 mag brighter than the red sequence (dashed red line, with the red sequence itself shown as the solid, red line). The dotted blue line indicates $z^{\prime}=23.5$; primary targets fainter than this are observed on multiple masks to increase exposure time. Six masks were designed for this cluster, and four have been observed. Large, filled points indicate galaxies already observed, while large open symbols are those allocated to masks that have not yet been observed. Some targets that lie outside the colour selection boundaries are included in the masks as 'fillers', once the mask is fully populated with priority targets.

where $n_{\mathrm{b}}$ and $n_{\mathrm{f}}$ represent the number of bright and faint objects, respectively, within $1 \mathrm{Mpc}$ of the cluster core allocated slits. This naturally downweights masks where there are insufficient faint galaxies in the final mask $(r \leq 1 \mathrm{Mpc})$. These are the most difficult to allocate as they must be allocated on every mask, and so negating this step naturally favours bright galaxies which need only be allocated to a single mask. The set of masks with the highest score from the 1000 realizations is used. As mentioned above, for cluster targets, one of the masks is typically band-shuffled, and so covers the central third of the GMOS field but with higher target density. This mask only contains bright galaxies, which would otherwise be underrepresented in the final sample due to geometry constraints. An example of the final target selection in colour-magnitude space for one of the clusters, SPT0546, is shown in Fig. 7. The figure shows the total sample from six GMOS masks, with galaxies already observed indicated with filled points.

For the massive group sample, the exquisite deep optical and X-ray data in COSMOS, CDFS, and SXDS make it possible to perform similar analysis on much lower mass haloes, following the GEEC2 strategy (Balogh et al. 2011). In particular, the highprecision photometric redshifts available improve target selection efficiency to a level comparable to that of the colour-selected cluster fields, without introducing significant bias. Instead of a straight sum of the number of galaxies, a weight $W$ is applied based on each galaxy's photometric redshift $\left(z_{\mathrm{ph}}\right)$, uncertainty $\left(\sigma_{\mathrm{zph}}\right)$, and its relation to the cluster redshift $\left(z_{\text {clus }}\right)$

$W= \begin{cases}1, & \text { if }\left|z_{\mathrm{ph}}-z_{\text {clus }}\right| \leq 2 \sigma_{\mathrm{zph}} \\ 0.5 & \text { if } 0.7 \leq z_{\mathrm{ph}} \leq 2.0 \\ 0.0 & \text { otherwise. }\end{cases}$ 
These weights are summed as in equation (1) to determine the best mask set.

Once the optimal set of primary target masks has been designed, the masks are examined to see where space for additional slits exists, and filler targets outside the primary sample are added. These extra targets are drawn from all galaxies in the merged $z^{\prime}$ and IRAC catalogue.

\section{SPECTROSCOPIC OBSERVATIONS AND DATA REDUCTION}

The spectroscopic data reduction is based on the IRAF tools provided by Gemini, via the Ureka distribution. A variance and data quality (DQ) plane are propagated through all the reduction steps. Table 4 describes when the spectroscopic data were acquired, as of midsemester 2017A. Forty-six independent masks have been observed, of which seven are in band-shuffle configuration. The weather condition constraints for this program are $\mathrm{CC} 70(<0.3$ mag extinction) and IQ70 $\left(0.5^{\prime \prime}-0.7^{\prime \prime}\right.$ in $z^{\prime}$ at zenith). However, most of these observations were carried out in Priority Visitor mode, where the visiting observers are able to choose when to execute their program, within an observing run that is generally longer than the allocated time. In many cases, this allowed us to take advantage of better conditions than planned for.

\subsection{Detrending and sky subtraction}

Bias frames observed close to each observation are combined and subtracted from all data. Bad pixel masks are created from the masks provided in the IRAF distribution, with additional bad pixels and columns identified from dark frames. Dark frames were observed in every semester, and these are also subtracted from the data. In semester 2016B, structure at the level of several hundred counts appeared in the GMOS-S detectors. This is correctable with bias subtraction, but the structure is variable from night to night. For these data, a unique bias frame is generated for every science frame, by linearly interpolating the two bias frames that bracket the science data, based on the time of observation.

Flat-field frames are interspersed with science frames, to allow accurate slit identification. The data are not flat-field corrected, however, as the statistical noise introduced by flat fielding is generally larger than any systematic effect it corrects. Cosmic ray rejection is performed using gemcrspec, which is a wrapper for the LA Cosmic routine (van Dokkum 2001).

The GMOS-S detector has three CCDs, each with a different QE as a function of wavelength. This is corrected using the gqecorr routine provided by Gemini, which generates a wavelength-dependent correction given a wavelength-calibrated frame (in our case an arc) and a flat-field frame. This correction is then applied to the wavelength-calibrated, sky-subtracted science frame. All our science data are taken in n\&s mode. Thus, sky subtraction is done simply by subtracting the science image from the corresponding sky image. We also produce a 'sky' spectrum by adding the two images. This is useful for checking the wavelength calibration (see below) and for distinguishing sky residuals from emission lines in our science data.

\subsection{Wavelength calibration}

Wavelength calibration is done using $\mathrm{CuAr}$ arc lamps, usually taken after a night's observing. At our low resolution, this lamp provides $\sim 10$ useful lines over the wavelength range $6200<\lambda<10700 \AA$.
The typical rms of the wavelength solution is $\sim 0.5 \AA$. All spectra (from both GMOS-S and GMOS-N) are linearized and rebinned to $3.91 \AA$ pixel $^{-1}$, and forced to span $5500<\lambda<10500 \AA$. In general, the wavelength calibration is not robust for $\lambda \lesssim 6000 \AA$, due to the lack of good arc lines at this resolution.

To account for simple shifts in the zero-point due to instrument flexure, we cross-correlate each sky spectrum with that of a reference slit, ideally chosen to have an accurate wavelength solution. The median shift for each mask is computed, and applied to the wavelength solution of that mask. Shifts are typically $<0.5$ pixels, though on occasion can be two or three times larger.

The final wavelength calibration is applied to the 'sky' spectra described above; all slits in a mask are then aligned in wavelength and displayed for a careful visual check of the wavelength solution.

\subsection{Charge diffusion correction}

Charge on the detector diffuses away from its original pixel, by a distance that increases with wavelength. This effect was described by Abraham et al. (2004). Because of the wavelength dependence, it is even more of a concern when using the Hamamatsu detectors, which have significant sensitivity beyond $1 \mu \mathrm{m}$. In our data, charge from bright sky lines spreads as far as 10 binned pixels, or 1". 6 . This is a serious problem in microshuffle mode, where the charge from the two nodded positions is typically separated by only one or two pixels. This results in sky residuals that do not subtract, in every slit. An example is shown in the top spectrum of Fig. 8. The same effect will also cause residuals in neighbouring slits when placed close together; however as this is much more difficult to correct for, and affects $\lesssim 10$ per cent of slits, we neglect it for now.

Because of our large data volume, we are able to implement an empirical correction that works well for most of the masks. First, we combine a set of sky-subtracted, wavelength-calibrated twodimensional science spectra. All spectra must have the same shuffle distance, which was either 38 or 40 pixels for the GMOS-S data. We need to consider each science slit, as well as its associated sky slit (produced by adding, rather than subtracting, data from the two nod positions). Slits with mean counts $>30$ within the wavelength range $9000<\lambda<9250 \AA$ are excluded; this was determined empirically as necessary to exclude some bad slits. Finally, we exclude data with $57000<$ MJD $<57100$ and $x_{\text {ccd }}<1000$ because, as we discuss below, these data are affected by an additional contribution. The selected slits are then combined with a weighted average, masking pixels with either a DQ flag, NaNs in either the science or sky frame, values of $<0$ in the sky frame or absolute values $>100$ in the science frame. The weights are the inverse of the mean sky counts within the wavelength range $9800<\lambda<10000 \AA$; this is a region of bright sky emission lines that produce the most detrimental effect on our science data. This produces a clean, high-S/N average of all our spectra; it includes the average science signal as well as any residuals not removed from n\&s sky subtraction. We also average the corresponding sky spectra, in exactly the same way.

The next step is to remove the average science signal from the average spectrum above. We do this by constructing an average of the sky-subtracted, wavelength-calibrated data from all bandshuffle slits. Since band-shuffle spectra are well separated on the detector, slit pairs are not contaminated by the charge diffusion, and 
Table 4. A log of all spectroscopic data obtained as of 2017 April (mid-semester 2017A). All data were acquired in Priority Visitor mode unless otherwise indicated in the final column.

\begin{tabular}{|c|c|c|c|c|c|c|}
\hline Target & Date & Mask & Band/Micro & $\begin{array}{l}\text { Telescope/ } \\
\text { Detector }\end{array}$ & $\begin{array}{l}\text { Integration } \\
\text { time (ks) }\end{array}$ & Notes \\
\hline SPT0205 & $\begin{array}{l}2014 \text { November } 16 \text { and } 18 \\
2016 \text { October } 29-30 \text { and November } 3 \\
2016 \text { October } 28-29\end{array}$ & $\begin{array}{l}\text { GS2014BLP001-06 } \\
\text { GS2016BLP001-02 } \\
\text { GS2016BLP001-09 }\end{array}$ & $\begin{array}{l}\text { Microshuffle } \\
\text { Microshuffle } \\
\text { Microshuffle }\end{array}$ & $\begin{array}{l}\text { GS/Ham } \\
\text { GS/Ham } \\
\text { GS/Ham }\end{array}$ & $\begin{array}{c}6.48 \\
10.8 \\
9.36\end{array}$ & \\
\hline SPT0546 & $\begin{array}{c}2014 \text { November } 15-16 \\
2014 \text { November } 17 \text { and } 19 \\
2015 \text { November } 20 \\
2015 \text { November } 21 \\
2016 \text { February } 10\end{array}$ & $\begin{array}{l}\text { GS2014BLP001-09 } \\
\text { GS2014BLP001-10 } \\
\text { GS2015BLP001-15 } \\
\text { GS2015BLP001-16 } \\
\text { GS2015BLP001-16 }\end{array}$ & $\begin{array}{l}\text { Microshuffle } \\
\text { Microshuffle } \\
\text { Microshuffle } \\
\text { Microshuffle } \\
\text { Microshuffle }\end{array}$ & $\begin{array}{l}\text { GS/Ham } \\
\text { GS/Ham } \\
\text { GS/Ham } \\
\text { GS/Ham } \\
\text { GS/Ham }\end{array}$ & $\begin{array}{l}5.76 \\
7.2 \\
7.92 \\
2.16 \\
14.4\end{array}$ & \\
\hline SpARCS0035 & $\begin{array}{l}2015 \text { November } 21 \\
2015 \text { November } 20 \\
2016 \text { October } 28 \\
2016 \text { October } 27\end{array}$ & $\begin{array}{l}\text { GS2015BLP001-05 } \\
\text { GS2015BLP001-06 } \\
\text { GS2016BLP001-01 } \\
\text { GS2016BLP001-07 }\end{array}$ & $\begin{array}{l}\text { Band shuffle } \\
\text { Microshuffle } \\
\text { Microshuffle } \\
\text { Microshuffle }\end{array}$ & $\begin{array}{l}\text { GS/Ham } \\
\text { GS/Ham } \\
\text { GS/Ham } \\
\text { GS/Ham }\end{array}$ & $\begin{array}{c}9.36 \\
7.2 \\
7.9 \\
10.8\end{array}$ & \\
\hline SpARCS1051 & $\begin{array}{l}2016 \text { February } 18 \text { and } 29 \\
2017 \text { April } 25 \\
2017 \text { April } 26\end{array}$ & $\begin{array}{l}\text { GN2016ALP004-03 } \\
\text { GN2017ALP004-08 } \\
\text { GN2017ALP004-07 }\end{array}$ & $\begin{array}{l}\text { Microshuffle } \\
\text { Microshuffle } \\
\text { Microshuffle }\end{array}$ & $\begin{array}{l}\text { GN/EEV } \\
\text { GN/Ham } \\
\text { GN/Ham }\end{array}$ & $\begin{array}{l}18.0 \\
12.0 \\
13.8\end{array}$ & Queue \\
\hline SpARCS1033 & $\begin{array}{l}2017 \text { April } 18 \\
2017 \text { April } 19 \\
2017 \text { April } 20\end{array}$ & $\begin{array}{l}\text { GN2017ALP004-01 } \\
\text { GN2017ALP004-02 } \\
\text { GN2017ALP004-03 }\end{array}$ & $\begin{array}{l}\text { Band shuffle } \\
\text { Microshuffle } \\
\text { Microshuffle }\end{array}$ & $\begin{array}{l}\text { GN/Ham } \\
\text { GN/Ham } \\
\text { GN/Ham }\end{array}$ & $\begin{array}{c}7.2 \\
10.08 \\
10.08\end{array}$ & \\
\hline SpARCS1034 & $\begin{array}{c}2017 \text { April } 24 \\
2017 \text { April } 12 \text { and } 27\end{array}$ & $\begin{array}{l}\text { GN2017ALP004-04 } \\
\text { GN2016ALP004-05 }\end{array}$ & $\begin{array}{l}\text { Band shuffle } \\
\text { Band shuffle }\end{array}$ & $\begin{array}{l}\text { GN/Ham } \\
\text { GN/Ham }\end{array}$ & $\begin{array}{c}4.3 \\
10.08\end{array}$ & \\
\hline SpARCS1616 & $\begin{array}{c}\text { 2016 June } 1 \\
\text { 2016 June } 2 \\
2017 \text { April } 18 \text { and } 27\end{array}$ & $\begin{array}{l}\text { GN2016ALP004-06 } \\
\text { GN2016ALP004-07 } \\
\text { GN2016ALP004-09 }\end{array}$ & $\begin{array}{l}\text { Microshuffle } \\
\text { Microshuffle } \\
\text { Microshuffle }\end{array}$ & $\begin{array}{l}\text { GN/EEV } \\
\text { GN/EEV } \\
\text { GN/Ham }\end{array}$ & $\begin{array}{l}14.4 \\
18.0 \\
17.28\end{array}$ & \\
\hline COSMOS-28 & 2016 January 30 & GN2015BLP004-03 & Microshuffle & GN/EEV & 18.0 & Queue \\
\hline COSMOS-63 & 2016 January 31 & GN2015BLP004-02 & Microshuffle & GN/EEV & 18.0 & Queue \\
\hline COSMOS-125 & $\begin{array}{l}2016 \text { January } 31 \\
2015 \text { February } 25\end{array}$ & $\begin{array}{l}\text { GS2016ALP001-02 } \\
\text { GS2015ALP001-02 }\end{array}$ & $\begin{array}{l}\text { Microshuffle } \\
\text { Microshuffle }\end{array}$ & $\begin{array}{l}\text { GS/Ham } \\
\text { GS/Ham }\end{array}$ & $\begin{array}{l}15.12 \\
12.25\end{array}$ & \\
\hline COSMOS-221 & $\begin{array}{l}2015 \text { February } 24 \\
2015 \text { February } 23 \\
2016 \text { February } 13\end{array}$ & $\begin{array}{l}\text { GS2015ALP001-01 } \\
\text { GS2014BLP001-05 } \\
\text { GS2016ALP001-01 }\end{array}$ & $\begin{array}{l}\text { Microshuffle } \\
\text { Microshuffle } \\
\text { Microshuffle }\end{array}$ & $\begin{array}{l}\text { GS/Ham } \\
\text { GS/Ham } \\
\text { GS/Ham }\end{array}$ & $\begin{array}{c}10.08 \\
5.04 \\
10.8\end{array}$ & \\
\hline SXDF49 & 2015 October 9 & GN2015BLP004-01 & Microshuffle & GN/EEV & 18.0 & Queue \\
\hline SXDF64 & 2014 November 17 & GS2014BLP001-08 & Microshuffle & GS/Ham & 7.2 & \\
\hline SXDF76 & 2014 November 15 & GS2014BLP001-02 & Microshuffle & GS/Ham & 5.76 & \\
\hline SXDF87 & 2014 November 15 & GS2014BLP001-07 & Microshuffle & GS/Ham & 8.64 & \\
\hline
\end{tabular}

the stack yields a two-dimensional spectrum of the average science data, free from residuals. For large enough samples like ours, where the input targets are identically selected, the average continuum from these masks should be a good match to the average continuum in the microshuffle masks, and we find this to be the case. This continuum can then be subtracted from the averaged microshuffle slit, after a renormalization to the average counts ${ }^{4}$ in the range 9000 $<\lambda<9280 \AA$. The result is a two-dimensional image that contains

\footnotetext{
${ }^{4}$ In practice, we average the absolute value of the three pixels near the peak of each of the positive- and negative-flux spectra; the average of a n\&s
} observation with perfect sky subtraction should be zero. 


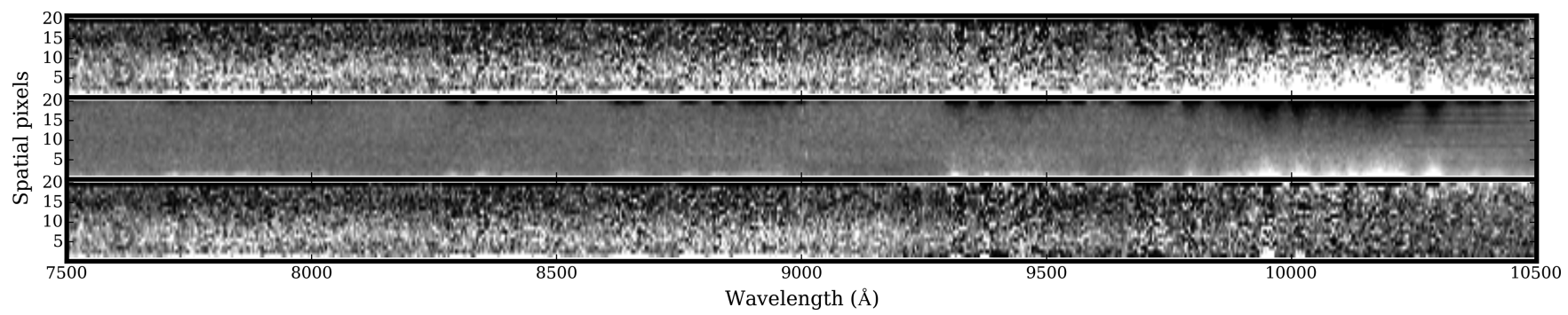

Figure 8. The top image shows the red end of a sky-subtracted, two-dimensional spectrum from a single slit in mask GS205ALP001-02. Strong residuals are evident at $\lambda>9500 \AA$ as positive flux near the bottom of the frame, and negative flux near the top. This is due to charge diffusion from the n\&s pair, as described in the text. To correct this, we create a template from a stack of sky-subtracted spectra, with continuum removed. This is shown in the middle panel, where the charge diffusion residuals are the only feature. After applying this correction to the data, we obtain the spectrum in the bottom panel. The grey scale is the same in all three images, ranging from -10 to +10 counts.

only the residuals due to charge diffusion, as shown in the middle spectrum of Fig. 8.

What remains is to subtract this 'master residual' from the data, after appropriate rescaling. The amplitude of the residual is expected to be directly proportional to the flux in the corresponding sky spectrum, since this light dominates over the object and uniformly fills the slit. Thus, we measure the mean signal of each column in the sky spectrum that corresponds to a given science slit. Pixels with DQ flags, or sky values $<0$ or $>5000$, are masked. The average is taken only of the four central rows, which are relatively free from science target flux. We take the ratio of this average to the average of the same pixels in the combined sky spectrum, and use this to scale the master residual image. Finally, this scaled image is subtracted from the science data, at $\lambda>8000 \AA$, where the effect is significant. The resulting science spectrum is free from these residuals, as shown in the bottom panel of Fig. 8. The subtracted flux is stored in a new extension labelled 'REDFIX'.

Three masks taken in early 2015 (two in COSMOS-221 and one for COSMOS-125) had to be dealt with separately. The charge diffusion here is larger than predicted by the simple scaling of the master residual described above. ${ }^{5}$ Having identified this, these images were excluded from the master residual described above. To deal with these frames, a similar process was followed, but using stacked residual frames in bins of date, $x_{\mathrm{ccd}}$ and $y_{\mathrm{ccd}}$. With significant trial and error to choose appropriate bin sizes, corrections were found that work reasonably well for these three masks.

The effect is also present on the EEV images from GMOS-N. It does not present as much of a problem here, as the detector sensitivity has died off by the time the effect becomes most problematic, beyond $\sim 9600 \AA$ A. Since we do not plan to obtain any band-shuffle masks with the EEV detectors on GMOS-N, a different procedure is required to remove the continuum from the residual stack. We take the band-shuffle continuum image described above and first rescale in the spatial direction to match the EEV pixel size, using a second-order spline. We then scale the intensity as a function of wavelength by the ratio of the EEV sensitivity relative to the Hamamatsu, using standard star observations. This serves to adequately model the average EEV continuum image, at least for $\lambda>8000 \AA$. This is subtracted from the combined microshuffle data as for the Hamamatsu observations, to produce an appropriate master residual frame.

\footnotetext{
${ }^{5}$ This may be related to a detector controller problem that was noted by Gemini a few weeks later, and which led to a much more severe charge smearing effect.
}

\subsection{Extraction and flux calibration}

We fully reduce each mask, including wavelength calibration, sky subtraction, and (where necessary) charge diffusion correction, and then median combine the slits using gemcombine, first rejecting the lowest and highest pixels. The reduced, two-dimensional image of each slit is $3^{\prime \prime}$ high, with an object spectrum at the top and an inverted spectrum at the bottom. We first compute an average spatial profile of the slit, by computing the median within $8000<\lambda<9750 \AA$, rejecting bad pixels using the DQ mask. We ignore the two pixels closest to either edge of the slit. This profile is fit with two Gaussian distributions, one with amplitude $A$ and the other with amplitude $-A$. Both are forced to have the same width, $\sigma$, and to be separated by a fixed amount given by the nod distance of 1 ". 45 . Next, we repeat the process for small intervals of wavelength (typically 250 or $500 \AA$ ), but keeping $\sigma$ fixed. Thus, we fit for only two parameters at each wavelength bin: the overall normalization, $A(\lambda)$, and the position of the bottom peak, $y(\lambda)$. Finally, we plot $y(\lambda)$ as a function of $\lambda$, and fit a polynomial to it with $2 \sigma$ rejection. The order of the polynomial, and the wavelength range of the fit, is determined interactively by the user. Typically, the order is $0-2$, and the fit is done over $6500<\lambda<9500 \AA$.

The spectral extraction is then a weighted sum of all pixels in a column (again omitting the top and bottom two pixels), where the weights are given by the double Gaussian function with vertical position at each wavelength given by the polynomial fit. Thus, most of the weight is given to the pixels at the centre of each spectrum. The amplitude is irrelevant, but the sign of the function ensures that the spectrum with negative flux is subtracted from the one with positive flux.

The extraction is made via a tool which shows the median extraction profile, the plot of $y(\lambda)$ versus $\lambda$ as well as the fit, the fit locations of both Gaussian peaks overlaid on the two-dimensional spectrum, and the spectrum extracted from this fit. Polynomial order, wavelength coverage, and binning are chosen interactively to ensure a good fit to each spectrum. The extraction parameters are stored in the header of the extracted spectrum.

Spectral flux standard observations are taken once each semester. The standards are reduced using the same pipeline described above, including the QE correction for GMOS-S. As these are not observed in n\&s mode, however, sky subtraction is done classically by defining a sky region adjacent to the source. The extracted spectrum is compared with tabulated values in the IRAF data base to generate a sensitivity function which is then applied to the extracted science data.

Bands of telluric absorption at $6850<\lambda / \AA \sim 6940$ ( $B$ band), $7550<\lambda / \AA<7710$ ( $A$ band), $8120<\lambda / \AA<8370$, and 


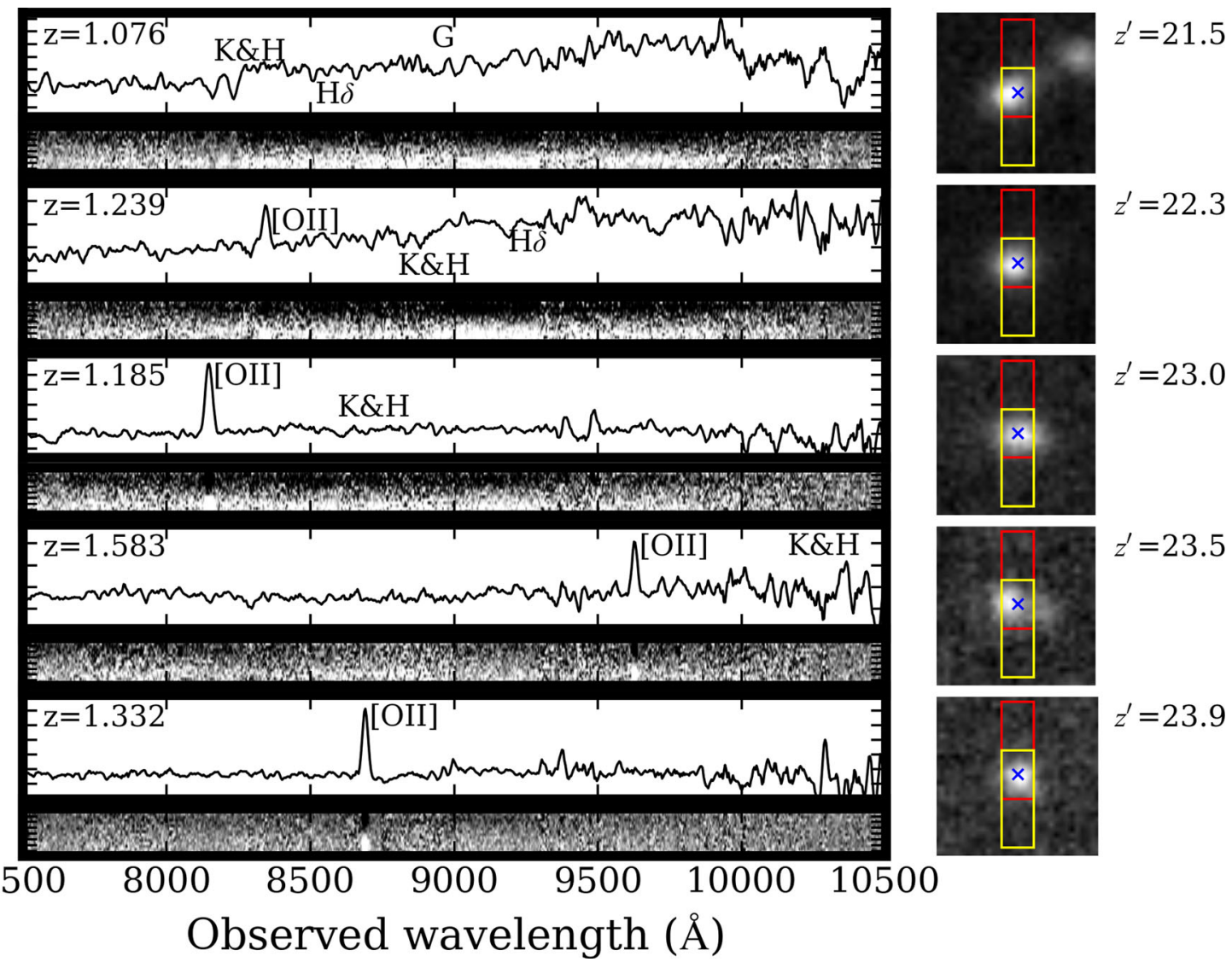

Figure 9. Sample data are shown for five galaxies in a range of $z^{\prime}$ magnitudes, indicated by the numbers on the right. Redshifts are given in the top left corner of each panel, and key identifiable spectral features are labelled (Calcium $\mathrm{H}$ and $\mathrm{K}, \mathrm{H} \delta$ and $G$-band absorption, and [O II] emission). The stamps on the right show the $z^{\prime}$-band image, scaled to the minimum and maximum counts within the subimage. The red and yellow rectangles show the predicted slit position in both nod positions. To the left are the one-dimensional (top) and two-dimensional (bottom) reduced spectra, after all reduction including sky subtraction, charge diffusion correction, and telluric/sensitivity correction (the latter applied only to the one-dimensional spectrum). All spectra shown here are from $7.2 \mathrm{ks}$ of exposure, and the one-dimensional spectra are convolved with a 5 pixel ( $20 \AA)$ boxcar filter. For the faintest two galaxies, the goal is to build up the $\mathrm{S} / \mathrm{N}$ by re-observing in multiple masks, with up to $54 \mathrm{ks}$ exposure by the end of the survey.

$8940<\lambda / \AA<9840$ are corrected using an IRAF package that crosscorrelates telluric features from our standard stars to compute a shift and scalefactor before subtracting from the data. This does not provide a perfect correction for the red features $(\lambda>8000 \AA)$, as these lines vary on short time-scales. Starting in 2016B, we have been including one bright star in each mask. By fitting templates to those stars we expect to derive a telluric correction which is applicable to all spectra in that mask. For earlier observations, we are exploring ways to use the existing data to derive improved corrections.

\subsection{Final spectra and science analysis}

In Fig. 9, we show some sample images and extracted one- and two-dimensional spectra for five targets after just $7.2 \mathrm{ks}$ exposure. A range of magnitudes are shown; the faintest galaxies $\left(z^{\prime}>23.5\right)$ will ultimately accrue up to $54 \mathrm{ks}$ of exposure by observing in multiple masks. The two-dimensional spectra shown are sky subtracted and corrected for charge diffusion. In these images, there is a positive- and negative-flux copies of the spectrum due to the $\mathrm{n} \& \mathrm{~s}$ technique. Note the spectrum is free from sky line residuals. The one-dimensional spectra are extracted as described in Section 3.4, including sensitivity calibration and preliminary telluric correction. Four of the spectra shown here show a strong [O II] emission line, clearly identifiable in both the one- and two-dimensional spectrum.
The top spectrum is a pure absorption line system, with the $\mathrm{Ca} \mathrm{H}+\mathrm{K}$ lines easily identifiable at $\lambda \sim 8200 \AA$.

Preliminary redshifts are being determined independently using the RUNZ code,${ }^{6}$ and an updated version of the DEEP2 specld pipeline (Newman et al. 2013). Future improvements will include adding rest-UV templates generated from our own data.

Stellar masses for the sample will be derived from SED fitting to multiwavelength photometry, including deep [3.6] $\mu \mathrm{m}$ imaging. For those clusters which currently lack sufficiently deep data, we have shown (Muzzin et al. 2012) that corrections for $M / L$ based on D4000 are sufficient to obtain masses to within a factor $\sim 2$ of those derived from SED fitting. This requires D4000 to be measured to within 20 per cent precision (corresponding to $\mathrm{S} / \mathrm{N} \sim 0.7 \mathrm{pixel}^{-1}$ ), which will be achievable for every galaxy for which we can get a redshift.

To measure the quiescent fraction, we need to classify our galaxies. This is best done using colour-colour diagrams spanning the rest-frame near-ultraviolet to NIR, which does an excellent job of separating dusty star-forming galaxies from truly passive galaxies (e.g. Muzzin et al. 2013a; Mok et al. 2013; Arnouts et al. 2013).

\footnotetext{
${ }^{6}$ http://www.physics.usyd.edu.au/scroom/runz/zguide.html
} 


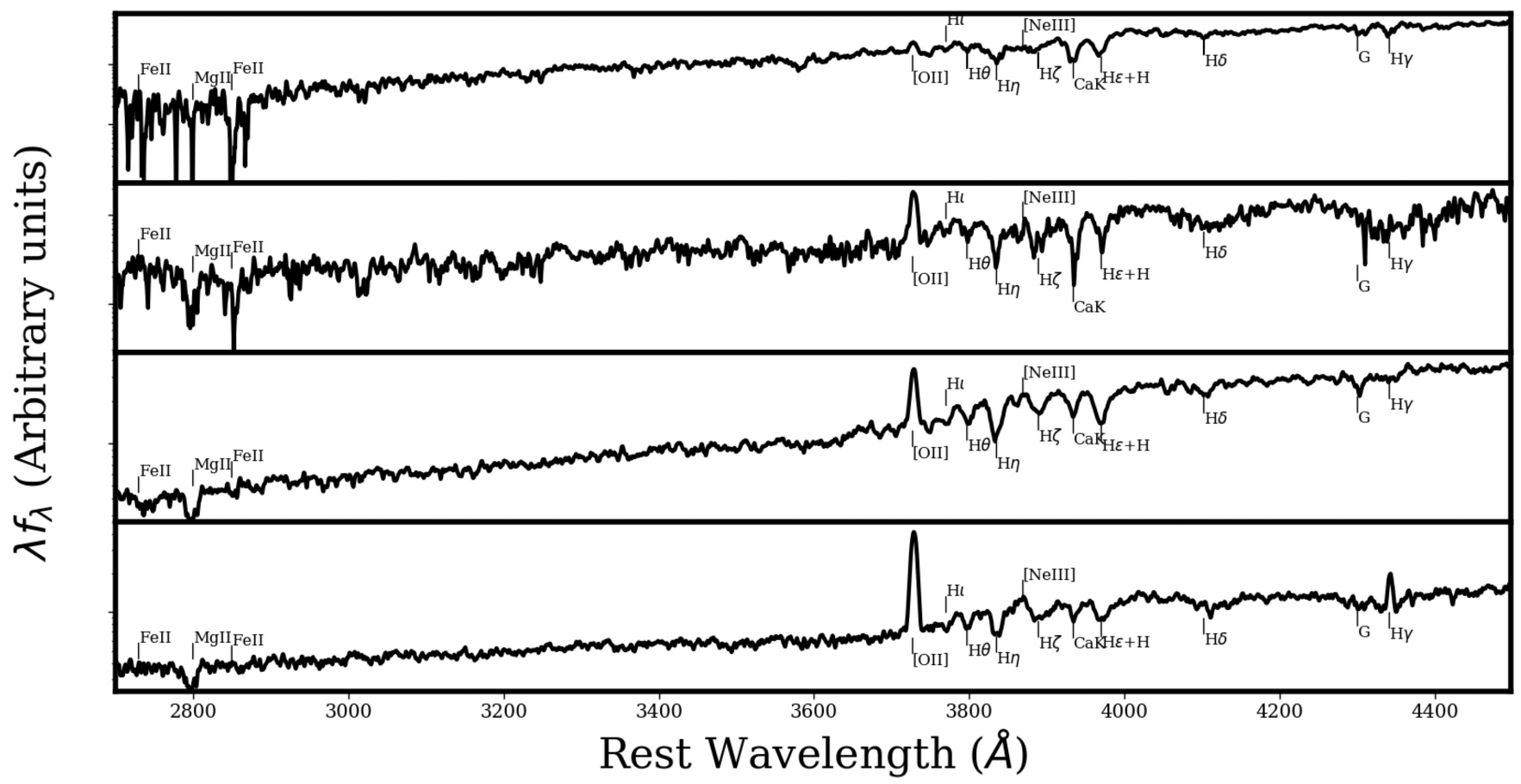

Figure 10. Averaged, resampled spectrum obtained by combining all of our $1.0<z<1.5$ spectra with good redshifts. From top to bottom, the panels show (a) bright, red; (b) faint, red; (c) bright, blue; and (d) faint, blue. The colour division is made based on D4000, and the bright/faint division is the subsample median.

This requires deep imaging spanning $u$ through $K$, which we have now obtained for most clusters in our sample (see Section 2.3.3).

Good age estimates for young- to intermediate-age populations can be obtained from absorption lines of $\mathrm{H} \delta$, Calcium $\mathrm{K}$ and $G$ band (Muzzin et al. 2014). While these lines cannot generally be measured reliably from individual spectra, we only need to stack $\sim 50$ galaxies to obtain $\mathrm{S} / \mathrm{N}>20$ per resolution element, sufficient to derive meaningful ages (e.g. Conroy, Graves \& van Dokkum 2014). Our sample size therefore allows us to calculate average spectra in bins of stellar mass, radius, and halo mass, and constrain the luminosity-weighted age to within $\sim 20$ per cent (Demarco et al. 2010a; Muzzin et al. 2014; Mok et al. 2014). As an early example of this, Fig. 10 shows a combined spectrum of all our $1.0<z<$ 1.5 spectra with good redshifts as of the end of the 2016A semester. We divide the sample into red and blue based on the D4000 index, with $D 4000>1.5$ being red. Within each subsample, we divide into two equal-sized luminosity bins. Each stack is comprised of about 40 galaxies. As well as the usual strong absorption $(\mathrm{Ca} \mathrm{H}+\mathrm{K}$, $G$ band) and emission ([O II]) lines, numerous weaker features are also detected at high $\mathrm{S} / \mathrm{N}$, including Fe II, $\mathrm{Mg}$ II, [Ne III], and higher order Balmer lines. By modelling the spectra with stellar population synthesis models, we will be able to determine ages and metallicities for galaxies as a function of their stellar mass and environment.

For the dynamical analysis, we expect to have $>50$ confirmed members in all systems but the groups, sufficient to keep within \pm 10 per cent the average bias of total mass estimates from their velocity dispersions (Biviano et al. 2006). More accurate, precise, and detailed dynamics can be determined when galaxies from several clusters are combined. For example, the 500 cluster members expected for our SpARCS sample alone are sufficient to constrain both the average total mass radial profile $M(r)$, and also the velocity anisotropy profile $\beta(r)$ of their member galaxies. Importantly, the sample size will be large enough to do this separately for the passive and star-forming populations, which are known to have different kinematics (e.g. Mohr, Geller \& Wegner 1996; Biviano et al. 1997). We will achieve this using the MAMPOSSt technique (Mamon, Biviano \& Boué 2013; Biviano et al. 2013), which breaks the intrinsic degeneracy between $M(r)$ and $\beta(r)$ in the Jeans equation. We will combine this analysis with the complementary caustic technique (Diaferio \& Geller 1997), to construct solutions that are independent of assumptions about dynamical equilibrium.

\section{SURVEY STATUS}

The total time awarded to GOGREEN was $438.3 \mathrm{~h}$, and a specific allocation is made each semester. Through mid-2017A, we have successfully executed $242 \mathrm{~h}$ of the $338.9 \mathrm{~h}$ allocated in this way, for a 71 per cent completion rate. 100 per cent of the required deep $z^{\prime}$-band imaging was obtained in the first two semesters, as planned; our spectroscopic program is now just over half complete. Our program was hit particularly hard by the very bad weather at Gemini-S during 2015, when only 18.3 of our $69 \mathrm{~h}$ allocation could be executed. This low completion rate has required an adjustment to our overall strategy. In early 2016, it was decided to postpone any further observations of the nine group targets in COSMOS and SXDS and focus on completing the massive cluster sample, to ensure an impactful program.

The other obstacle was the delayed deployment of Hamamatsu detectors on Gemini-N, which meant that observations of our high-redshift northern targets were pushed towards the end of the program. For these reasons we requested, and were granted, a program extension of two semesters, to the end of 2018A.

Analysis of the spectroscopic data acquired so far shows we are reaching our target S/N. In Fig. 11, we show the projected, final $\mathrm{S} / \mathrm{N}$ for all objects for which we have some existing data. The $\mathrm{S} / \mathrm{N}$ is measured over $8250-8750 \AA$, the most relevant range 


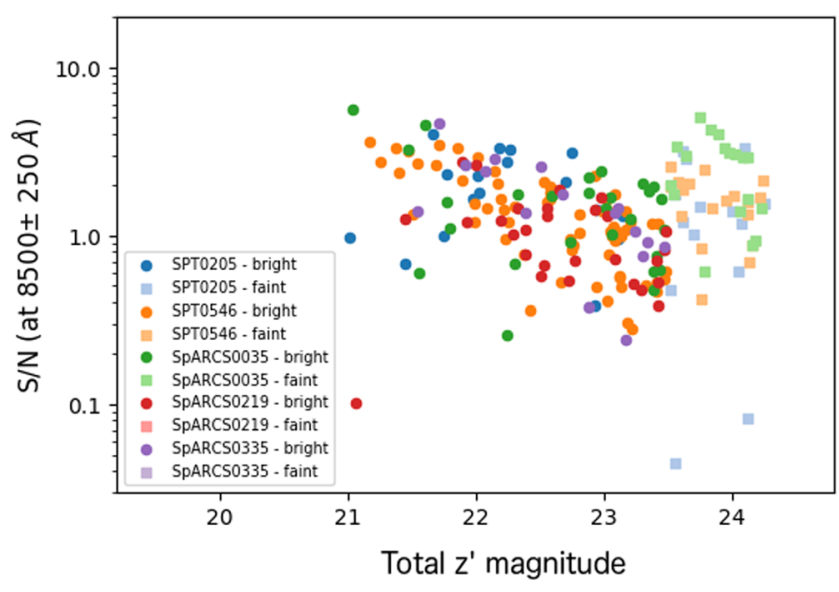

Figure 11. The projected final $\mathrm{S} / \mathrm{N}$ per $\AA$, measured in a $500 \AA$ window centred on $8500 \AA$, is shown for every primary target spectrum obtained as of semester 2016B, as a function of total $z^{\prime}$-band magnitude. Each symbol colour corresponds to a different mask. The measured $\mathrm{S} / \mathrm{N}$ for each spectrum is scaled to its predicted value at the end of the survey, assuming $3 \mathrm{~h}$ exposures for $z^{\prime}<23.5$ galaxies, and $15 \mathrm{~h}$ exposures for $z^{\prime}>23.5$ galaxies.

for redshift determination. The measurements are scaled from the current exposure time for each spectrum, assuming a final exposure of $3 \mathrm{~h}$ for $z^{\prime}<23.5$ galaxies and $15 \mathrm{~h}$ for $z^{\prime}>23.5$ galaxies. This shows that we expect to achieve $\mathrm{S} / \mathrm{N} \gtrsim 0.7$ per $\AA$, or $>3$ per resolution element $(\sim 20 \AA)$ for $\sim 80$ per cent of our targets. This is consistent with our proposal objectives.

In Fig. 12, we show two examples of colour-magnitude diagrams, with coloured points indicating the spectroscopic sample. GOGREEN is extending existing spectroscopy in these systems as expected, down to [3.6] $\mu \mathrm{m}<22.5$, with high completeness. Neither of these fields has achieved its full $\mathrm{S} / \mathrm{N}$ at this point; thus, redshift completeness will increase by the end of the survey.

In the right-hand panels of Fig. 12, we show the spatial distribution of the galaxies in these two clusters. Unlike GCLASS, GOGREEN does not focus on the dense core of the cluster, but aims for a more even sampling beyond a $\sim 1 \mathrm{Mpc}$ radius. However, the restriction to one GMOS field of view, and the need to keep the full-wavelength coverage in most spectra, means the spectral sample is extended in one dimension more than the other. In clusters which do not have existing spectroscopy of the core as these examples do, we execute at least one band-shuffled mask to ensure a high sampling of relatively bright galaxies near the centre.

\subsection{Public data release}

GOGREEN has committed to release the first data products no later than one year after the end of the survey. The final data release will include at least reduced spectra, reduced GMOS images, and catalogues of redshifts, GMOS photometry, and advanced data products including line indices and photometric redshifts. The GCLASS and GEEC2 data will also be provided as part of this release. Details will be available in a forthcoming paper devoted to the data release.

\section{CONCLUSION}

The GOGREEN survey is a Large Program on Gemini North and South, using a large allocation of time $(>400 \mathrm{~h})$ to construct an unprecedented sample of homogeneously selected galaxy spectroscopy in 21 galaxy clusters and groups at $1.0<z<1.5$. The targets are chosen to span a wide range in halo mass, such that they correspond to the progenitors of the massive clusters and groups that are well studied at $z \sim 0$. The red sensitivity of the Hamamatsu detectors, coupled with the n\&s mode, allows good quality spectra to be obtained at $\lambda<1.05 \mu \mathrm{m}$, and with this we probe galaxies of all types with $M_{*} \gtrsim 10^{10.3} \mathrm{M}_{\odot}$ over the whole redshift range. We provide a thorough description of the GMOS data reduction, and the specific challenges associated with the Hamamatsu detectors. In addition to the deep spectroscopy, we have acquired deep multiwavelength photometry, spanning ugrizYJK and Spitzer [3.6] $\mu \mathrm{m}$ in most systems, over and beyond the full spectroscopic field of view. With these data, we will investigate the role of environment in galaxy evolution, at an epoch when the overall galaxy population is forming stars at a much higher rate than today. The data also enable an analysis of cluster dynamics and stellar content over a wide range in halo mass. We anticipate the sample will have high legacy value, including a sample of $\sim 600$ field galaxies with spectroscopic redshifts down to faint magnitudes $z^{\prime}<24.25$. The first public data release will occur within one year of the survey completion.

\section{ACKNOWLEDGEMENTS}

We extend our thanks and appreciation to Gemini, and the Gemini communities, for supporting this Large Program. We also thank Gemini for their support of junior observers on several of our observing runs.

This research is supported by the following grants: NSERC Discovery grants (MLB and LCP); Universidad Andrés Bello internal project grants DI-651-15/R and DI-18-17/RG (JN); NSF grants AST-1517815 and AST-1211358 (GHR), AST-1517863 (GW) and AST-1518257 (MCC); NASA, through grants AR14310.001 and GO-12945.001-A (GHR), GO-13306, GO-13677, GO-13747 and GO-13845/14327 (GW), AR-13242 and AR-14289 (MCC) and HST-GO-14734 (AW) from the Space Telescope Science Institute, which is operated by AURA, Inc., under NASA contract NAS 5-26555; STFC (SLM); the BASAL Center for Astrophysics and Associated Technologies (CATA), and FONDECYT grant no. 1130528 (RD); the European Research Council under FP7 grant number 340519 (RFJvdB); the National Research Foundation of South Africa (DGG); Chandra grant AR6-17014B (AFF); and FONDECYT postdoctoral research grant no. 3160375 (PC). AW was also supported by a Caltech-Carnegie Fellowship, in part through the Moore Center for Theoretical Cosmology and Physics at Caltech. GHR also acknowledges the hospitality and financial support of the Max-Planck-Institute for Extraterrestrial Physics, the European Southern Observatory and the International Space Sciences Institute as well as the hospitality of the Max-Planck-Institute for Astronomy and Hamburg Observatory.

This paper includes data gathered with the Gemini Observatory, which is operated by the Association of Universities for Research in Astronomy, Inc., under a cooperative agreement with the NSF on behalf of the Gemini partnership: the National Science Foundation (United States), the National Research Council (Canada), CONICYT (Chile), Ministerio de Ciencia, Tecnologa e Innovacin Productiva (Argentina), and Ministrio da Ciłncia, Tecnologia e Inovao (Brazil); the 6.5 metre Magellan Telescopes located at Las Campanas Observatory, Chile; the Canada-France-Hawaii Telescope (CFHT) which is operated by the National Research Council of Canada, the Institut National des Sciences de l'Univers of the Centre National de la Recherche Scientifique of France, and the University of Hawaii; MegaPrime/MegaCam, a joint project of CFHT and CEA/DAPNIA; Subaru Telescope, which is operated 
SPT0546

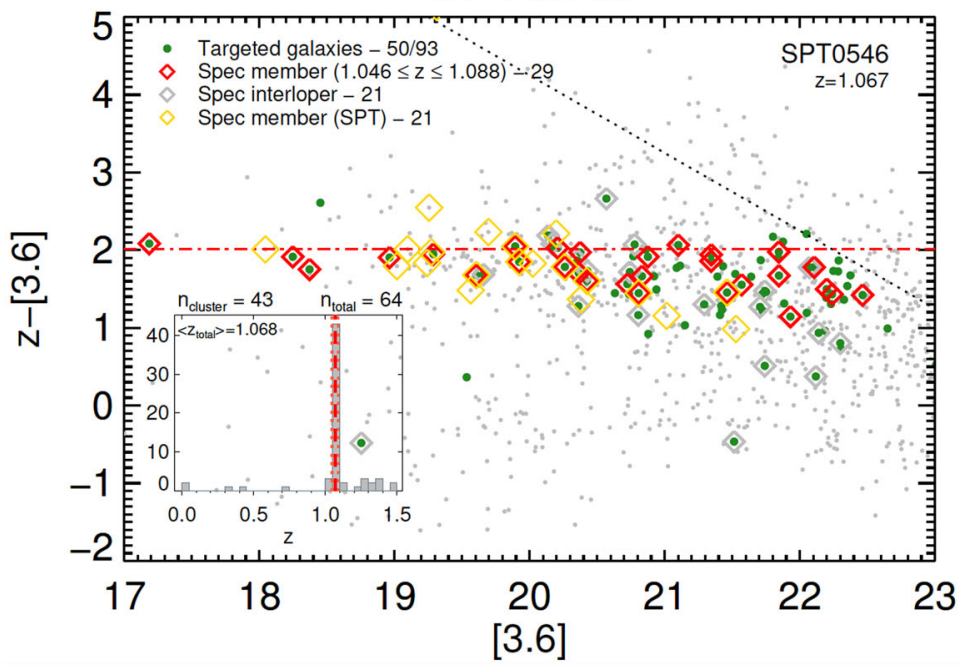

SpARCS1634

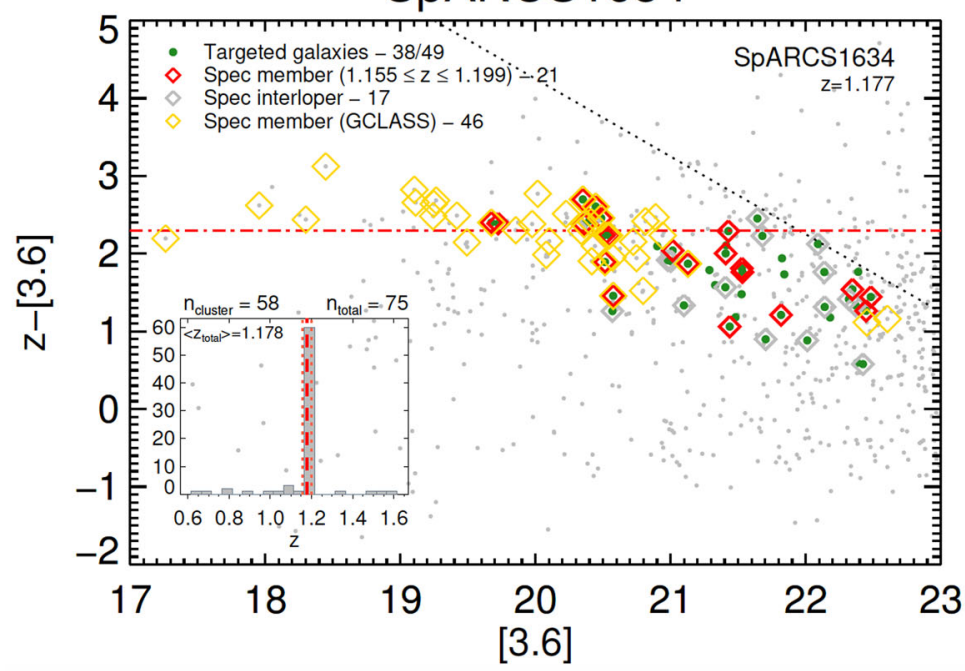

SPT0546
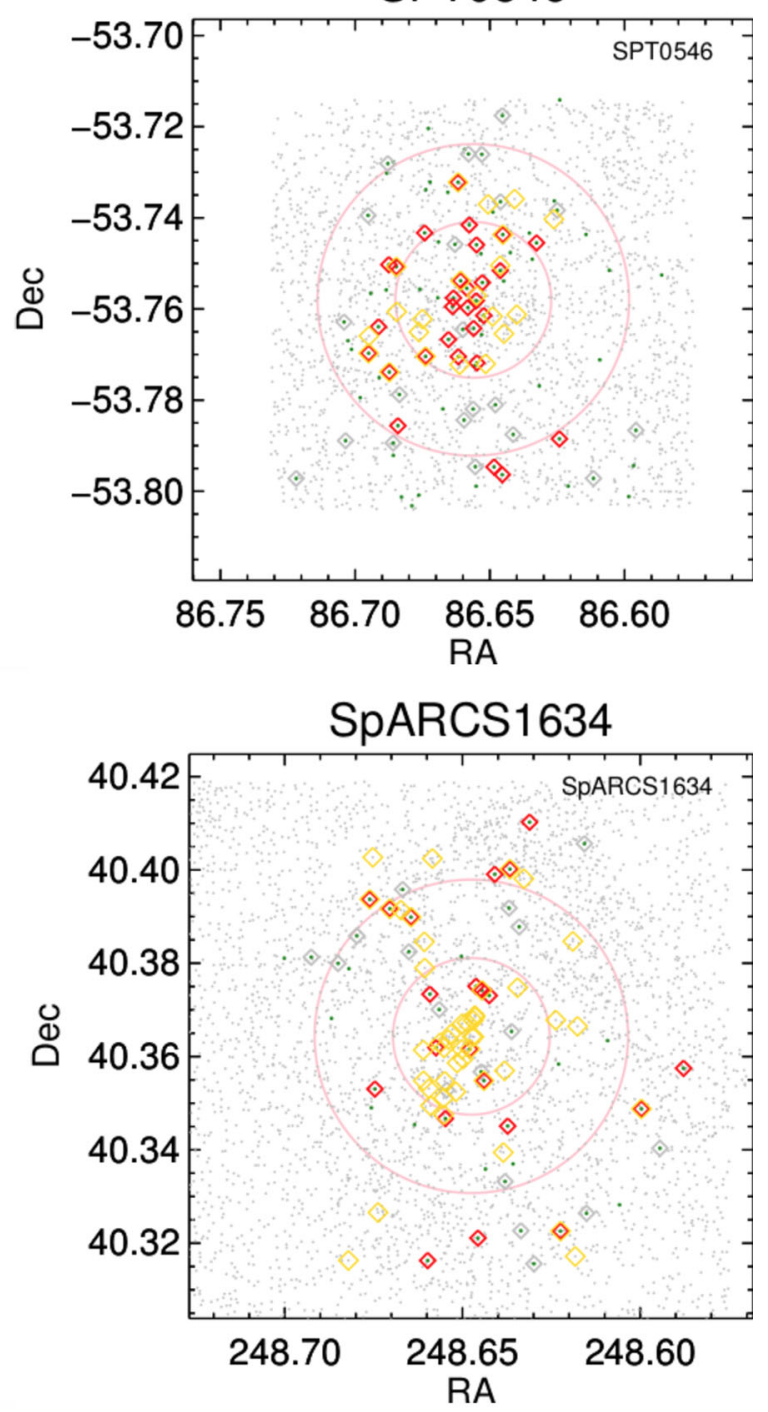

Figure 12. Preliminary colour-magnitude diagrams (left) and spatial distribution of the spectroscopy (right) are shown for two of our clusters, as labelled. The red horizontal line shows the adopted red sequence position, and the dotted black line represents the $z^{\prime}$ target selection limit. Red diamonds indicate new spectroscopic members from our GOGREEN spectroscopy, while yellow diamonds are existing redshifts from GCLASS and SPT. Grey diamonds are GOGREEN galaxies with redshifts confirmed to be non-members, while green circles are galaxies that do not yet have a reliable redshift. Neither field has achieved its full $\mathrm{S} / \mathrm{N}$ at this point in the survey, so redshift completeness will increase. The inset on the left plot shows the redshift histogram with an estimated number of cluster members and mean redshift indicated. The spatial plots on the right show how the spectroscopic members are distributed relative to 0.5 and $1.0 \mathrm{Mpc}$ radii around the field centre.

by the National Astronomical Observatory of Japan; and the ESO Telescopes at the La Silla Paranal Observatory under programme ID 097.A-0734.

\section{REFERENCES}

Abraham R. G. et al., 2004, AJ, 127, 2455

Aharonian F. A. et al., 2017, ApJ, 837, L15

Alexander D. M., Hickox R. C., 2012, New Astron. Rev., 56, 93

Arnouts S. et al., 2013, A\&A, 558, A67

Balogh M. L., Babul A., Patton D. R., 1999a, MNRAS, 307, 463

Balogh M. L., Morris S. L., Yee H. K. C., Carlberg R. G., Ellingson E., 1999b, ApJ, 527, 54

Balogh M. L., Navarro J. F., Morris S. L., 2000, ApJ, 540, 113

Balogh M. L., McCarthy I. G., Bower R. G., Eke V. R., 2008, MNRAS, 385, 1003
Balogh M. L. et al., 2011, MNRAS, 412, 2303

Balogh M. L. et al., 2014, MNRAS, 443, 2679

Balogh M. L. et al., 2016, MNRAS, 456, 4364

Bayliss M. B. et al., 2016, ApJS, 227, 3

Behroozi P. S., Wechsler R. H., Conroy C., 2013, ApJ, 770, 57

Benson B. A. et al., 2013, ApJ, 763, 147

Berrier J. C., Stewart K. R., Bullock J. S., Purcell C. W., Barton E. J., Wechsler R. H., 2009, ApJ, 690, 1292

Bertin E., Arnouts S., 1996, A\&AS, 117, 393

Bialek J. J., Evrard A. E., Mohr J. J., 2001, ApJ, 555, 597

Biviano A., Girardi M., 2003, ApJ, 585, 205

Biviano A., Katgert P., 2004, A\&A, 424, 779

Biviano A., Poggianti B. M., 2009, A\&A, 501, 419

Biviano A., Katgert P., Mazure A., Moles M., den Hartog R., Perea J., Focardi P., 1997, A\&A, 321, 84

Biviano A., Murante G., Borgani S., Diaferio A., Dolag K., Girardi M., 2006, A\&A, 456, 23 
Biviano A. et al., 2013, A\&A, 558, A1

Biviano A., van der Burg R. F. J., Muzzin A., Sartoris B., Wilson G., Yee H. K. C., 2016, A\&A, 594, A51

Boselli A. et al., 2016, A\&A, 587, A68

Bower R. G., Benson A. J., Malbon R., Helly J. C., Frenk C. S., Baugh C. M., Cole S., Lacey C. G., 2006, MNRAS, 370, 645

Bower R. G., Benson A. J., Crain R. A., 2012, MNRAS, 422, 2816

Bradač M., Allen S. W., Treu T., Ebeling H., Massey R., Morris R. G., von der Linden A., Applegate D., 2008, ApJ, 687, 959

Brodwin M. et al., 2010, ApJ, 721, 90

Bruzual G., Charlot S., 2003, MNRAS, 344, 1000

Bullock J. S., Kravtsov A. V., Weinberg D. H., 2001, ApJ, 548, 33

Butcher H., Oemler A., 1984, ApJ, 285, 426

Carlberg R. G., Yee H. K. C., Ellingson E., Abraham R., Gravel P., Morris S., Pritchet C. J., 1996, ApJ, 462, 32

Cavagnolo K. W., Donahue M., Voit G. M., Sun M., 2008, ApJ, 683, L107

Clowe D., Bradač M., Gonzalez A. H., Markevitch M., Randall S. W., Jones C., Zaritsky D., 2006, ApJ, 648, L109

Conroy C., Graves G. J., van Dokkum P. G., 2014, ApJ, 780, 33

Davé R., Finlator K., Oppenheimer B. D., 2012, MNRAS, 421, 98

De Lucia G. et al., 2004, ApJ, 610, L77

De Lucia G., Boylan-Kolchin M., Benson A. J., Fontanot F., Monaco P., 2010, MNRAS, 406, 1533

De Lucia G., Weinmann S., Poggianti B. M., Aragón-Salamanca A., Zaritsky D., 2012, MNRAS, 423, 1277

Dehnen W., McLaughlin D. E., 2005, MNRAS, 363, 1057

Demarco R. et al., 2010a, ApJ, 725, 1252

Demarco R. et al., 2010b, ApJ, 711, 1185

Diaferio A., Geller M. J., 1997, ApJ, 481, 633

Dressler A., 1980, ApJ, 236, 351

Einasto J., Kaasik A., Saar E., 1974, Nature, 250, 309

Eisenhardt P. R. et al., 2004, ApJS, 154, 48

Ellingson E., Lin H., Yee H. K. C., Carlberg R. G., 2001, ApJ, 547, 609

Fabian A. C. et al., 2000, MNRAS, 318, L65

Fassbender R. et al., 2014, A\&A, 568, A5

Fillingham S. P., Cooper M. C., Wheeler C., Garrison-Kimmel S., BoylanKolchin M., Bullock J. S., 2015, MNRAS, 454, 2039

Fillingham S. P., Cooper M. C., Pace A. B., Boylan-Kolchin M., Bullock J. S., Garrison-Kimmel S., Wheeler C., 2016, MNRAS, 463, 1916

Finoguenov A. et al., 2007, ApJS, 172, 182

Finoguenov A. et al., 2010, MNRAS, 403, 2063

Foley R. J. et al., 2011, ApJ, 731, 86

Foltz R. et al., 2015, ApJ, 812, 138

Fossati M. et al., 2017, ApJ, 835, 153

Galametz A. et al., 2013, ApJS, 206, 10

Genel S. et al., 2014, MNRAS, 445, 175

George M. R. et al., 2011, ApJ, 742, 125

Gilbank D. G., Balogh M. L., 2008, MNRAS, 385, L116

Giodini S. et al., 2009, ApJ, 703, 982

Gladders M. D., Yee H. K. C., 2000, AJ, 120, 2148

Glazebrook K., Bland-Hawthorn J., 2001, PASP, 113, 197

Gonzalez A. H., Zaritsky D., Zabludoff A. I., 2007, ApJ, 666, 147

Gonzalez A. H., Sivanandam S., Zabludoff A. I., Zaritsky D., 2013, ApJ, 778,14

Haiman Z., Mohr J. J., Holder G. P., 2001, ApJ, 553, 545

Haines C. P. et al., 2015, ApJ, 806, 101

Hansen S. M., Sheldon E. S., Wechsler R. H., Koester B. P., 2009, ApJ, 699, 1333

Henriques B. M. B., White S. D. M., Thomas P. A., Angulo R., Guo Q., Lemson G., Springel V., Overzier R., 2015, MNRAS, 451, 2663

Hirschmann M., De Lucia G., Wilman D., Weinmann S., Iovino A., Cucciati O., Zibetti S., Villalobos Á., 2014, MNRAS, 444, 2938

Hoekstra H., Mahdavi A., Babul A., Bildfell C., 2012, MNRAS, 427, 1298 Hou A. et al., 2013, MNRAS, 435, 1715

Jee M. J., Mahdavi A., Hoekstra H., Babul A., Dalcanton J. J., Carroll P., Capak P., 2012, ApJ, 747, 96

Kereš D., Katz N., Weinberg D. H., Davé R., 2005, MNRAS, 363, 2

Kravtsov A. V., Nagai D., Vikhlinin A. A., 2005, ApJ, 625, 588
Lacy M. et al., 2005, ApJS, 161, 41

Lapi A., Cavaliere A., 2009, ApJ, 695, L125

Leauthaud A., Tinker J., Behroozi P. S., Busha M. T., Wechsler R. H., 2011, ApJ, 738, 45

Leauthaud A. et al., 2012, ApJ, 746, 95

Lidman C. et al., 2012, MNRAS, 427, 550

Lilly S. J., Carollo C. M., Pipino A., Renzini A., Peng Y., 2013, ApJ, 772, 119

Lin Y.-T., Brodwin M., Gonzalez A. H., Bode P., Eisenhardt P. R. M., Stanford S. A., Vikhlinin A., 2013, ApJ, 771, 61

Lonsdale C. J. et al., 2003, PASP, 115, 897

Lotz J. M. et al., 2013, ApJ, 773, 154

Mamon G. A., Biviano A., Boué G., 2013, MNRAS, 429, 3079

Marchesini D. et al., 2014, ApJ, 794, 65

Martini P. et al., 2013, ApJ, 768, 1

Mauduit J.-C. et al., 2012, PASP, 124, 714

McGee S. L., Balogh M. L., Bower R. G., Font A. S., McCarthy I. G., 2009, MNRAS, 400, 937

McGee S. L., Balogh M. L., Wilman D. J., Bower R. G., Mulchaey J. S., Parker L. C., Oemler, A., Jr, 2011, MNRAS, 413, 996

McGee S. L., Bower R. G., Balogh M. L., 2014, MNRAS, 442, L105

McNamara B. R., Nulsen P. E. J., 2007, ARA\&A, 45, 117

Merluzzi P. et al., 2013, MNRAS, 429, 1747

Mohr J. J., Geller M. J., Wegner G., 1996, AJ, 112, 1816

Mohr J. J., Mathiesen B., Evrard A. E., 1999, ApJ, 517, 627

Mok A. et al., 2013, MNRAS, 431, 1090

Mok A. et al., 2014, MNRAS, 438, 3070

Muzzin A., Wilson G., Lacy M., Yee H. K. C., Stanford S. A., 2008, ApJ, 686, 966

Muzzin A. et al., 2009, ApJ, 698, 1934

Muzzin A. et al., 2012, ApJ, 746, 188

Muzzin A. et al., 2013a, ApJ, 777, 18

Muzzin A. et al., 2013b, ApJS, 206, 8

Muzzin A. et al., 2014, ApJ, 796, 65

Nanayakkara T. et al., 2016, ApJ, 828, 21

Nantais J. B., Rettura A., Lidman C., Demarco R., Gobat R., Rosati P., Jee M. J., 2013, A\&A, 556, A112

Nantais J. B. et al., 2016, A\&A, 592, A161

Natarajan P., Kneib J.-P., Smail I., 2002, ApJ, 580, L11

Navarro J. F., Frenk C. S., White S. D. M., 1997, ApJ, 490, 493

Newman J. A. et al., 2013, ApJS, 208, 5

Noble A. G., Webb T. M. A., Muzzin A., Wilson G., Yee H. K. C., van der Burg R. F. J., 2013, ApJ, 768, 118

Noble A. G., Webb T. M. A., Yee H. K. C., Muzzin A., Wilson G., van der Burg R. F. J., Balogh M. L., Shupe D. L., 2016, ApJ, 816, 48

Peng Y. et al., 2010, ApJ, 721, 193

Peng Y.-j., Lilly S. J., Renzini A., Carollo M., 2012, ApJ, 757, 4

Pfrommer C., Enßlin T. A., 2004, A\&A, 413, 17

Poggianti B. M. et al., 2009, ApJ, 693, 112

Postman M. et al., 2012, ApJS, 199, 25

Puchwein E., Sijacki D., Springel V., 2008, ApJ, 687, L53

Quadri R. F., Williams R. J., Franx M., Hildebrandt H., 2012, ApJ, 744, 88

Randall S. W., Markevitch M., Clowe D., Gonzalez A. H., Bradač M., 2008, ApJ, 679, 1173

Rosati P. et al., 2014, The Messenger, 158, 48

Rudnick G. et al., 2009, ApJ, 700, 1559

Rudnick G. H., Tran K.-V., Papovich C., Momcheva I., Willmer C., 2012, ApJ, 755, 14

Sand D. J. et al., 2012, ApJ, 746, 163

Sanders D. B. et al., 2007, ApJS, 172, 86

Schawinski K. et al., 2014, MNRAS, 440, 889

Sehgal N. et al., 2011, ApJ, 732, 44

Snyder G. F. et al., 2012, ApJ, 756, 114

Stalder B. et al., 2013, ApJ, 763, 93

Stanford S. A., Gonzalez A. H., Brodwin M., Gettings D. P., Eisenhardt P. R. M., Stern D., Wylezalek D., 2014, ApJS, 213, 25

Tanaka M., Kodama T., Arimoto N., Okamura S., Umetsu K., Shimasaku K., Tanaka I., Yamada T., 2005, MNRAS, 362, 268 
Taranu D. S., Hudson M. J., Balogh M. L., Smith R. J., Power C., Oman K. A., Krane B., 2014, MNRAS, 440, 1934

Tinker J. L., Wetzel A. R., 2010, ApJ, 719, 88

Tozzi P., Norman C., 2001, ApJ, 546, 63

Trayford J. W. et al., 2015, MNRAS, 452, 2879

van de Voort F., Bahé Y. M., Bower R. G., Correa C. A., Crain R. A., Schaye J., Theuns T., 2017, MNRAS, 466, 3460

van den Bosch F. C., Aquino D., Yang X., Mo H. J., Pasquali A., McIntosh

D. H., Weinmann S. M., Kang X., 2008, MNRAS, 387, 79

van der Burg R. F. J. et al., 2013, A\&A, 557, A15

van der Burg R. F. J., Muzzin A., Hoekstra H., Wilson G., Lidman C., Yee H. K. C., 2014, A\&A, 561, A79

van Dokkum P. G., 2001, PASP, 113, 1420

Vikhlinin A., Kravtsov A., Forman W., Jones C., Markevitch M., Murray S. S., Van Speybroeck L., 2006, ApJ, 640, 691

Voit G. M., Bryan G. L., 2001, Nature, 414, 425

Vollmer B., Marcelin M., Amram P., Balkowski C., Cayatte V., Garrido O., 2000, A\&A, 364, 532

Wang L., Steinhardt P. J., 1998, ApJ, 508, 483

Weinmann S. M., Lisker T., Guo Q., Meyer H. T., Janz J., 2011, MNRAS, 416, 1197

Weinmann S. M., Pasquali A., Oppenheimer B. D., Finlator K., Mendel J. T., Crain R. A., Macciò A. V., 2012, MNRAS, 426, 2797

Wetzel A. R., Tinker J. L., Conroy C., van den Bosch F. C., 2013, MNRAS, 432, 336

White S. D. M. et al., 2005, A\&A, 444, 365

Williams R. J., Quadri R. F., Franx M., van Dokkum P., Labbé I., 2009, ApJ, 691, 1879

Wilman D. J., Balogh M. L., Bower R. G., Mulchaey J. S., Oemler A., Carlberg R. G., Morris S. L., Whitaker R. J., 2005, MNRAS, 358, 71

Wilson G. et al., 2009, ApJ, 698, 1943

Yee H. K. C., Ellingson E., Carlberg R. G., 1996, ApJS, 102, 269

Zeimann G. R. et al., 2013, ApJ, 779, 137

${ }^{1}$ Department of Physics and Astronomy, University of Waterloo, Waterloo, Ontario, ON N2L 3G1, Canada

${ }^{2}$ South African Astronomical Observatory, P.O. Box 9, Observatory 7935 Cape Town, South Africa

${ }^{3}$ Centre for Space Research, North-West University, Potchefstroom 2520 Cape Town, South Africa

${ }^{4}$ Department of Physics and Astronomy, York University, 4700 Keele Street, Toronto, Ontario, ON MJ3 1P3, Canada

${ }^{5}$ Department of Physics and Astronomy, The University of Kansas, 1251 Wescoe Hall Drive, Lawrence, KS 66045, USA

${ }^{6}$ Department of Physics and Astronomy, University of California, Irvine, 4129 Frederick Reines Hall, Irvine, CA 92697, USA
${ }^{7}$ Australian Astronomical Observatory, 105 Delhi Road, North Ryde, NSW 2113, Australia

${ }^{8}$ INAF - Osservatorio Astronomico di Trieste, via G. B. Tiepolo 11, I-34143 Trieste, Italy

${ }^{9}$ Departamento de Astronomía, Universidad de Concepción, Casilla 160-C, Concepción, 0000-0003-3921-2177 Región del Biobio, Chile

${ }^{10}$ School of Physics and Astronomy, University of Birmingham, Edgbaston, Birmingham B15 2TT, England

${ }^{11}$ Departamento de Ciencias Físicas, Universidad Andres Bello, Fernandez Concha 700, Las Condes 7591538, Santiago, Región Metropolitana, Chile

${ }^{12}$ MIT Kavli Institute for Astrophysics and Space Research, 70 Vassar St, Cambridge, MA 02109, USA

${ }^{13}$ Department of Astronomy and Astrophysics, University of Toronto 50 St. George Street, Toronto, Ontario M5S 3H4, Canada

${ }^{14}$ Department of Physics and Astronomy, University of California Riverside, 900 University Avenue, Riverside, CA 92521, USA

${ }^{15}$ European Southern Observatory, Alonso de Cordova 3107, Vitacura, Casilla 19001, Santiago de Chile, Chile

${ }^{16}$ Laboratoire AIM, IRFU/Service d'Astrophysique - CEA/DRF - CNRS

- Université Paris Diderot, Bât. 709, CEA-Saclay, F-91191 Gif-sur-Yvette Cedex, France

${ }^{17}$ Steward Observatory and Department of Astronomy, University of Arizona, Tucson, AZ 85719, USA

${ }^{18}$ Observatorio Astronómico de Córdoba (UNC) and Instituto de Astronomía, Laprida 925 Teórica y Experimetal (CONICET-UNC), Córdoba, Argentina

${ }^{19}$ Institute for Computational Cosmology, Durham University, South Road, Durham DH1 3LE, UK

${ }^{20}$ Center for Extragalactic Astronomy, Durham University, South Road, Durham DH1 3LE, UK

${ }^{21}$ Department of Physics, University of Helsinki, Gustaf Hällströmin katu 2a, FI-00014 Helsinki, Finland

${ }^{22}$ Max-Planck-Institut für extraterrestrische Physik, Giessenbachstr. 1, Garching, D-85741, Germany

${ }^{23}$ Department of Physics and Astronomy, McMaster University, Hamilton ON L8S 4M1, Canada

${ }^{24}$ IPAC, Caltech, KS 314-6, 1200 E. California Blvd, Pasadena, CA 91125, USA

${ }^{25}$ Carnegie Observatories, Pasadena, CA, USA

${ }^{26}$ TAPIR, California Institute of Technology, Pasadena, 91125-0001 CA, USA

${ }^{27}$ Department of Physics, University of California, Davis, 95616 CA, USA

This paper has been typeset from a $\mathrm{T}_{\mathrm{E}} \mathrm{X} / \mathrm{L} \mathrm{T} \mathrm{E} \mathrm{X}$ file prepared by the author. 